TRANSACTIONS OF THE

AMERICAN MATHEMATICAL SOCIETY

Volume 356, Number 1, Pages 219-243

S 0002-9947(03)03116-7

Article electronically published on August 25, 2003

\title{
VASSILIEV INVARIANTS FOR BRAIDS ON SURFACES
}

\author{
JUAN GONZÁLEZ-MENESES AND LUIS PARIS
}

\begin{abstract}
We show that Vassiliev invariants separate braids on a closed oriented surface, and we exhibit a universal Vassiliev invariant for these braids in terms of chord diagrams labeled by elements of the fundamental group of the surface.
\end{abstract}

\section{Definitions And Statements}

1.1. Introduction. Vassiliev knot invariants were introduced by V. A. Vassiliev ([17, 18]; see also 6], 2]), and they have been generalized to several other knotlike objects, such as links, braids, tangles, string links, knotted graphs, etc. The purpose of this paper is to consider Vassiliev invariants of braids on surfaces, and to extend some well-known results on Vassiliev invariants of Artin braids to the case of braids on surfaces.

Our study of Vassiliev invariants is inspired by Papadima's work [13] on Vassiliev invariants for Artin braids with values in $\mathbb{Z}$. However, the presence of the fundamental group of the surface changes the analysis substantially. Anyway, the Vassiliev theory for braids on surfaces, set forth in this paper, appears to be a natural generalization of the corresponding theory for Artin braids.

1.2. Braids and singular braids on surfaces. Throughout this paper $M$ will denote a closed, orientable surface of genus $g \geq 1$, and $\mathcal{P}=\left\{P_{1}, \ldots, P_{n}\right\}$ a set of $n$ distinct points in $M$. Define a $n$-braid based at $\mathcal{P}$ to be a collection $b=\left(b_{1}, \ldots, b_{n}\right)$ of disjoint smooth paths in $M \times[0,1]$, called strings of $b$, such that the $i$-th string $b_{i}$ runs monotonically in $t \in[0,1]$ from the point $\left(P_{i}, 0\right)$ to some point $\left(P_{j}, 1\right), P_{j} \in \mathcal{P}$.

An isotopy in this context is a deformation through braids (which fixes the ends). Multiplication of braids is defined by concatenation, generalizing the construction of the fundamental group. The isotopy classes of braids with this multiplication form the group $B_{n}(M, \mathcal{P})$, called the braid group with $n$ strings on $M$ based at $\mathcal{P}$. Note that the group $B_{n}(M, \mathcal{P})$ does not depend, up to isomorphism, on the set $\mathcal{P}$ of points, but only on the cardinality $n=|\mathcal{P}|$. So we may write $B_{n}(M)$ in place of $B_{n}(M, \mathcal{P})$.

In the same way as Artin braid groups have been extended to singular braid monoids ([6], [1]), one can extend the braid group $B_{n}(M)$ to $S B_{n}(M)$, the monoid of singular braids with $n$ strings on $M$. The strings of a singular braid are now

Received by the editors November 7, 2000 and, in revised form, May 20, 2002.

2000 Mathematics Subject Classification. Primary 20F36; Secondary 57M27, 57N05.

Key words and phrases. Braid, surface, Vassiliev invariant, finite type invariant.

The first author was supported in part by DGESIC-PB97-0723, by BFM2001-3207 and by the European network TMR Sing. Eq. Diff. et Feuill. 
allowed to intersect transversely, but only in finitely many double points, called singular points.

As with braids, isotopy is a deformation through singular braids (which fixes the ends), and multiplication is by concatenation. Note that the isotopy classes of singular braids form a monoid and not a group: the singular braids with one or more singular points being non-invertible.

1.3. Vassiliev invariants and Vassiliev filtration. An invariant of braids on $M$ with values in an abelian group $A$, is a set-mapping $v: B_{n}(M) \rightarrow A$. Just as for knots and Artin braids, one can extend $v$ to singular braids by using the recursive rule

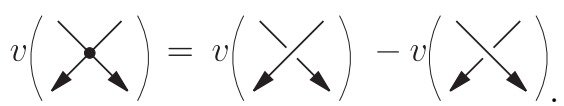

The picture on the left-hand side represents a small neighborhood of a singular point in a singular braid. Those on the right-hand side represent the braids which are obtained from the previous one by resolution of that singular point. That is, we modify the first braid inside the neighborhood of the singular point, in a positive and a negative way, to obtain two singular braids having one less singular point.

Let $d$ be an integer. A Vassiliev invariant of type $d$ is an invariant $v$ such that $v(b)=0$ for every singular braid $b$ with more than $d$ singular points.

There is an equivalent definition of a Vassiliev invariant, in terms of the so-called Vassiliev filtration. First, consider the group $\operatorname{ring} \mathbb{Z}\left[B_{n}(M)\right]$. We can define a map

$$
\eta: S B_{n}(M) \longrightarrow \mathbb{Z}\left[B_{n}(M)\right]
$$

which "resolves" all the singular points of a given braid, with the corresponding signs. That is,

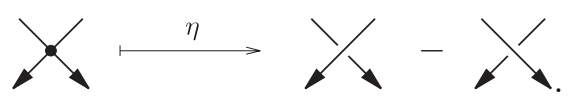

This map is a well-defined multiplicative morphism. Remark that a singular braid with $d$ singular points is mapped to an alternate sum of $2^{d}$ non-singular braids, each one having coefficient +1 or -1 depending on the sign of its corresponding resolutions.

Let $S_{d} B_{n}(M)$ denote the set of isotopy classes of singular braids with $d$ singular points. We denote by $V_{d}$ the $\mathbb{Z}$-module generated by $\eta\left(S_{d} B_{n}(M)\right)$. One can easily verify that $V_{d}$ is a (two-sided) ideal of $\mathbb{Z}\left[B_{n}(M)\right]$ and that we have the inclusions $V_{d+1} \subset V_{d}$ and $V_{d_{1}} V_{d_{2}}=V_{d_{1}+d_{2}}$, for all $d_{1}, d_{2}, d \in \mathbb{N}$. We have then obtained a filtration

$$
\mathbb{Z}\left[B_{n}(M)\right]=V_{0} \supset V_{1} \supset V_{2} \supset \cdots,
$$

which is called the Vassiliev filtration of $\mathbb{Z}\left[B_{n}(M)\right]$.

The definition of a Vassiliev invariant in terms of the Vassiliev filtration is as follows. One can extend any invariant $v: B_{n}(M) \rightarrow A$ by linearity to a morphism of $\mathbb{Z}$-modules $v: \mathbb{Z}\left[B_{n}(M)\right] \rightarrow A$. Note that the previous extension of $v$ to singular braids can also be expressed by $v(b)=v(\eta(b))$, for $b \in S B_{n}(M)$. Then, $v$ is a Vassiliev invariant of type $d$ if and only if it vanishes on $V_{d+1}$. Therefore, the set of Vassiliev invariants of type $d$ with values in $A$ is equal to

$$
\operatorname{Hom}_{\mathbb{Z}}\left(\mathbb{Z}\left[B_{n}(M)\right] / V_{d+1}, A\right) .
$$


1.4. Statements. We have two goals in this paper. The first one is to show that Vassiliev invariants separate braids on surfaces, that is, to prove the following.

Theorem 1.1. Given two non-equivalent braids $b$ and $c$ on $M$, there exist an integer $N \geq 1$ and a Vassiliev invariant $v_{N}$ of type $N$ such that $v_{N}(b) \neq v_{N}(c)$. Moreover, $v_{N}$ can be chosen to take values in $\mathbb{Z}$.

This result is known to hold for Artin braids ([3], 10], 13]), but it is still a conjecture for knots. Actually, Theorem 1.1 is a corollary of the following theorem.

Theorem 1.2. Let $\left\{V_{d}\right\}_{d=1}^{\infty}$ be the Vassiliev filtration of $\mathbb{Z}\left[B_{n}(M)\right]$. Then

(1) $\bigcap_{d=0}^{\infty} V_{d}=\{0\}$, and

(2) $V_{d} / V_{d+1}$ is a free $\mathbb{Z}$-module for all $d \geq 0$.

Indeed, if Theorem 1.2 holds, then, given two non-equivalent braids $b, c \in B_{n}(M)$, there exists an integer $N$ such that $b-c \notin V_{N+1}$. Then we can take $v_{N}$ to be the canonical projection from $\mathbb{Z}\left[B_{n}(M)\right]$ to $\mathbb{Z}\left[B_{n}(M)\right] / V_{N+1}$. In addition, if $V_{d} / V_{d+1}$ is a free $\mathbb{Z}$-module for all $d$, then

$$
\mathbb{Z}\left[B_{n}(M)\right] / V_{N+1} \simeq\left(\mathbb{Z}\left[B_{n}(M)\right] / V_{1}\right) \oplus\left(V_{1} / V_{2}\right) \oplus \cdots \oplus\left(V_{N} / V_{N+1}\right)
$$

is also a free $\mathbb{Z}$-module, so we can obviously compose the above projection with a map from $\mathbb{Z}\left[B_{n}(M)\right] / V_{N+1}$ to $\mathbb{Z}$, in such a way that the image of $b-c$ is non-zero. Therefore, our first goal will be achieved by proving Theorem 1.2 .

Our second goal is to define a universal Vassiliev invariant for $B_{n}(M)$ which generalizes the notion of chord diagrams for Artin braids. Recall that a chord diagram is a diagram made of $n$ vertical lines and a finite number of horizontal segments, called chords, connecting the lines. An M-labeled chord diagram is a chord diagram such that each chord is labeled by an element of $\pi_{1}(M)$ (see Figure 1). Note that the set of $M$-labeled chord diagrams is equipped with a multiplication defined by concatenation. The free $\mathbb{Z}$-module generated by the chord diagrams is a $\mathbb{Z}$-algebra which can be identified with $\mathbb{Z}\left[t_{i, j, \gamma}\right]$, the free non-commutative $\mathbb{Z}$-algebra freely generated by the $t_{i, j, \gamma}$, where $i, j \in\{1, \ldots, n\}, i \neq j, \gamma \in \pi_{1}(M)$, and where $t_{i, j, \gamma}=t_{j, i, \gamma^{-1}}$ (see Figure 1).
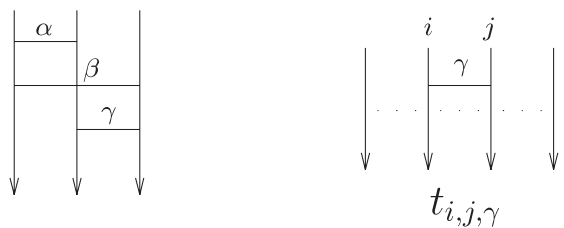

Figure 1. An $M$-labeled chord diagram and the generator $t_{i, j, \gamma}$.

We denote by $\mathcal{A}_{n}$ the quotient $\mathbb{Z}$-algebra obtained from $\mathbb{Z}\left[t_{i, j, \gamma}\right]$ by imposing the relations

$$
\begin{array}{ll}
-\left[t_{i, j, \gamma}, t_{k, l, \delta}\right]=0, & \text { for all distinct } i, j, k, l \in\{1, \ldots, n\} \\
\text { - }\left[t_{i, j, \gamma}, t_{j, k, \delta}+t_{i, k,(\gamma \delta)}\right]=0, & \text { and all } \gamma, \delta \in \pi_{1}(M), \\
& \text { for all distinct } i, j, k \in\{1, \ldots, n\} \\
& \text { and all } \gamma, \delta \in \pi_{1}(M),
\end{array}
$$

and we denote by $\widehat{\mathcal{A}}_{n}$ its natural completion. 
Note that the symmetric group $\Sigma_{n}$ acts on $\pi_{1}(M)^{n}$ by permuting coordinates, so we can consider the induced semi-direct product $H_{n}=\pi_{1}(M)^{n} \rtimes \Sigma_{n}$. In addition, it is straightforward to show that $H_{n}$ acts on $\widehat{\mathcal{A}}_{n}$, defining the semi-direct product $\widehat{\mathcal{A}}_{n} \rtimes \mathbb{Z}\left[H_{n}\right]$. The action is defined by the following relations:

- $\sigma t_{i, j, \gamma} \sigma^{-1}=t_{\sigma(i), \sigma(j), \gamma}, \quad$ for all $\sigma \in \Sigma_{n}$,

- $\mu(k) t_{i, j, \gamma} \mu(k)^{-1}=t_{i, j, \gamma}, \quad$ for all $\mu \in \pi_{1}(M)$ and all $k \neq i, j$,

- $\mu(i) t_{i, j, \gamma} \mu(i)^{-1}=t_{i, j,(\mu \gamma)}$, for all $\mu \in \pi_{1}(M)$,

where $\mu(i)=(1, \ldots, 1, \stackrel{(i)}{\mu}, 1, \ldots, 1) \in \pi_{1}(M)^{n}$. Note that one also has the following relation:

$$
\mu(j) t_{i, j, \gamma} \mu(j)^{-1}=\mu(j) t_{j, i, \gamma^{-1}} \mu(j)^{-1}=t_{j, i,\left(\mu \gamma^{-1}\right)}=t_{i, j,\left(\gamma \mu^{-1}\right)} .
$$

The $\mathbb{Z}$-algebra $\widehat{\mathcal{A}}_{n} \rtimes \mathbb{Z}\left[H_{n}\right]$ carries the filtration induced by that of $\widehat{\mathcal{A}}_{n}$, so its associated graded algebra is $\mathcal{A}_{n} \rtimes \mathbb{Z}\left[H_{n}\right]$. We also have $\operatorname{gr}_{V} \mathbb{Z}\left[B_{n}(M)\right]=$ $\bigoplus_{d=0}^{\infty}\left(V_{d} / V_{d+1}\right)$. Our second main result will be

Theorem 1.3. There exists a homomorphism of $\mathbb{Z}$-modules $u: \mathbb{Z}\left[B_{n}(M)\right] \rightarrow$ $\widehat{\mathcal{A}}_{n} \rtimes \mathbb{Z}\left[H_{n}\right]$ such that the corresponding graded map

$$
\operatorname{gr} u: \operatorname{gr}_{V} \mathbb{Z}\left[B_{n}(M)\right] \longrightarrow \mathcal{A}_{n} \rtimes \mathbb{Z}\left[H_{n}\right]
$$

is an isomorphism of graded $\mathbb{Z}$-algebras.

We end this section by showing why $u$ is called a universal Vassiliev invariant for $B_{n}(M)$.

Corollary 1.4. Every Vassiliev invariant of $B_{n}(M)$ factors through $u$ in a unique way.

Proof. By Theorem $\left[1.2\right.$, we know that $\mathbb{Z}\left[B_{n}(M)\right] / V_{N+1}$ is a free $\mathbb{Z}$-module for all $N \geq 0$; hence, $\mathbb{Z}\left[B_{n}(M)\right] \simeq\left(\mathbb{Z}\left[B_{n}(M)\right] / V_{N+1}\right) \oplus V_{N+1}$. Recall that

$$
\operatorname{gr}_{V} \mathbb{Z}\left[B_{n}(M)\right]=\bigoplus_{d=0}^{\infty}\left(V_{d} / V_{d+1}\right) \simeq\left(\mathbb{Z}\left[B_{n}(M)\right] / V_{N+1}\right) \oplus\left(\bigoplus_{d>N}\left(V_{d} / V_{d+1}\right)\right)
$$

Now, since gr $u$ is an isomorphism, we conclude that, for all $N \geq 0, \mathcal{A}_{n}^{(\leq N)} \rtimes \mathbb{Z}\left[H_{n}\right]$ is also a free $\mathbb{Z}$-module. Therefore,

$$
\widehat{\mathcal{A}}_{n} \rtimes \mathbb{Z}\left[H_{n}\right] \simeq\left(\mathcal{A}_{n}^{(\leq N)} \rtimes \mathbb{Z}\left[H_{n}\right]\right) \oplus\left(\widehat{\mathcal{A}}_{n}^{(>N)} \rtimes \mathbb{Z}\left[H_{n}\right]\right)
$$

and

$$
\mathcal{A}_{n} \rtimes \mathbb{Z}\left[H_{n}\right] \simeq\left(\mathcal{A}_{n}^{(\leq N)} \rtimes \mathbb{Z}\left[H_{n}\right]\right) \oplus\left(\mathcal{A}_{n}^{(>N)} \rtimes \mathbb{Z}\left[H_{n}\right]\right) .
$$

Every Vassiliev invariant $v \in \operatorname{Hom}_{\mathbb{Z}}\left(\mathbb{Z}\left[B_{n}(M)\right] / V_{N+1}, A\right)$ can then be seen as a linear map from $\operatorname{gr}_{V} \mathbb{Z}\left[B_{n}(M)\right]$ to $A$ which vanishes on $\bigoplus_{d>N}\left(V_{d} / V_{d+1}\right)$. Via gru, this means that $v$ is a linear map from $\mathcal{A}_{n} \rtimes \mathbb{Z}\left[H_{n}\right]$ to $A$, which vanishes on $\mathcal{A}_{n}^{(>N)} \rtimes \mathbb{Z}\left[H_{n}\right]$. Therefore, if $v$ is a Vassiliev invariant of type $N$, it can be lifted in a unique way to a linear map $\widehat{v}: \widehat{\mathcal{A}}_{n} \rtimes \mathbb{Z}\left[H_{n}\right] \rightarrow A$, which satisfies $v=\widehat{v} \circ u$. 


\section{VASSILIEV INVARIANTS SEPARATE BRAIDS}

Our strategy for proving Theorem 1.2 is the following. In a first subsection, we introduce some ideal $J$ of $\mathbb{Z}\left[B_{n}(M)\right]$ given by its generators, and we prove that $V_{d}=J^{d}$ for all $d \geq 0$. In a second subsection, we consider an exact sequence $1 \rightarrow K_{n} \rightarrow B_{n}(M) \rightarrow H_{n} \rightarrow 1$, and we prove that $J^{d}$ is equal in some sense to $I\left(K_{n}\right)^{d} \otimes \mathbb{Z}\left[H_{n}\right]$, where $I\left(K_{n}\right)$ denotes the augmentation ideal of $K_{n}$. In a third subsection, we prove that $K_{n}$ can be expressed as an iterated semi-direct product of free groups (of infinite rank). Finally, in the fourth subsection, we use the results of the previous ones to prove Theorem 1.2 .

2.1. The Vassiliev filtration coincides with the $J$-adic filtration. The aim of this subsection is to introduce an ideal $J$ of $\mathbb{Z}\left[B_{n}(M)\right]$ defined by its generators, and to show that the Vassiliev filtration coincides with the $J$-adic filtration (i.e., $V_{d}=J^{d}$ for all $d \in \mathbb{N}$ ).

We begin by explaining our "visualization" of (singular) braids, and by exhibiting generators for $S B_{n}(M)$.

We represent the surface $M$ as a polygon of $4 g$ sides which are identified as shown in Figure 2.

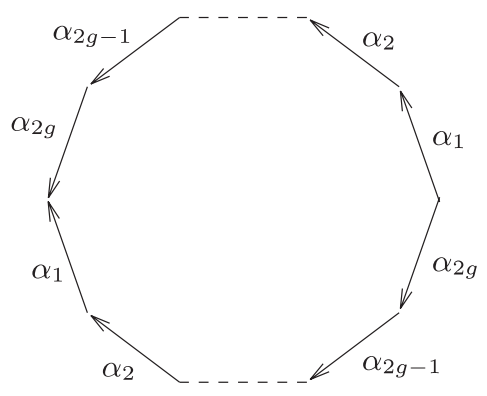

FIgURE 2. A representation of the surface $M$.

We draw braids over $M$ in this polygon, as if we looked at the cylinder $M \times$ $[0,1]$ from above; that is, we project the braid onto $M \times\{0\}$. As for the planar representations of knots, we see over- and under-crossings, and we can always move our braid via a suitable isotopy to avoid triple crossing points in the projection. See Figure 3 for an example.

Now, for every $i \in\{1, \ldots, n\}$ and every $r \in\{1, \ldots, 2 g\}$, we define the braid $a_{i, r}$ as follows. All the strings of $a_{i, r}$ are trivial except the $i$-th one, which goes through the $r$-th wall as shown in Figure4. It goes upwards if $r$ is odd and downwards if $r$ is even.

We also define, for all $j=1, \ldots, n-1$, the braid $\sigma_{j}$ as follows. All the strings of $\sigma_{j}$ are trivial except the $j$-th one and the $(j+1)$-th one. The $j$-th string goes from $\left(P_{j}, 0\right)$ to $\left(P_{j+1}, 1\right)$ and the $(j+1)$-th string goes from $\left(P_{j+1}, 0\right)$ to $\left(P_{j}, 1\right)$, according to Figure 4. Note that $\sigma_{1}, \ldots, \sigma_{n-1}$ are the classical generators of the braid group $B_{n}$ of the disc.

It is easy to show that $\left\{a_{i, r} ; i=1, \ldots, n, r=1, \ldots, 2 g\right\} \cup\left\{\sigma_{1}, \ldots, \sigma_{n-1}\right\}$ is a generator set for $B_{n}(M)$. Actually, there is no need to include $a_{i, r}$ if $i \geq 2$, but it is better for our purposes. One can find in [9] a presentation for $B_{n}(M)$ which involves these generators. 

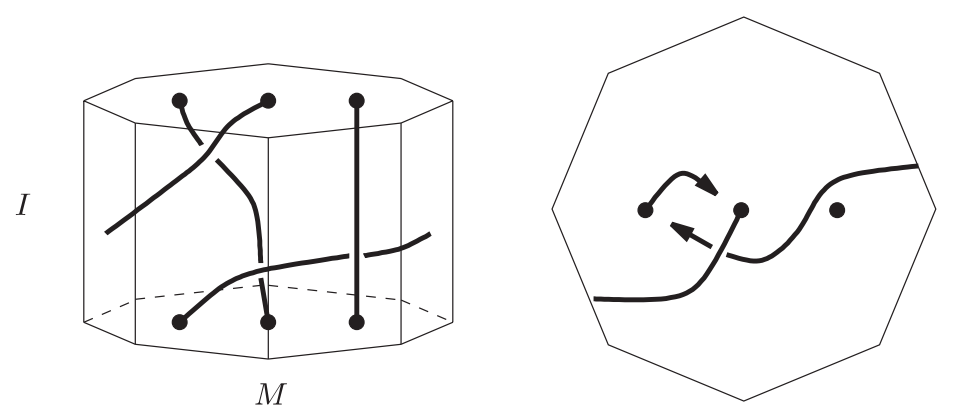

Figure 3. A braid with 3 strings on a surface of genus 2: two different viewpoints.

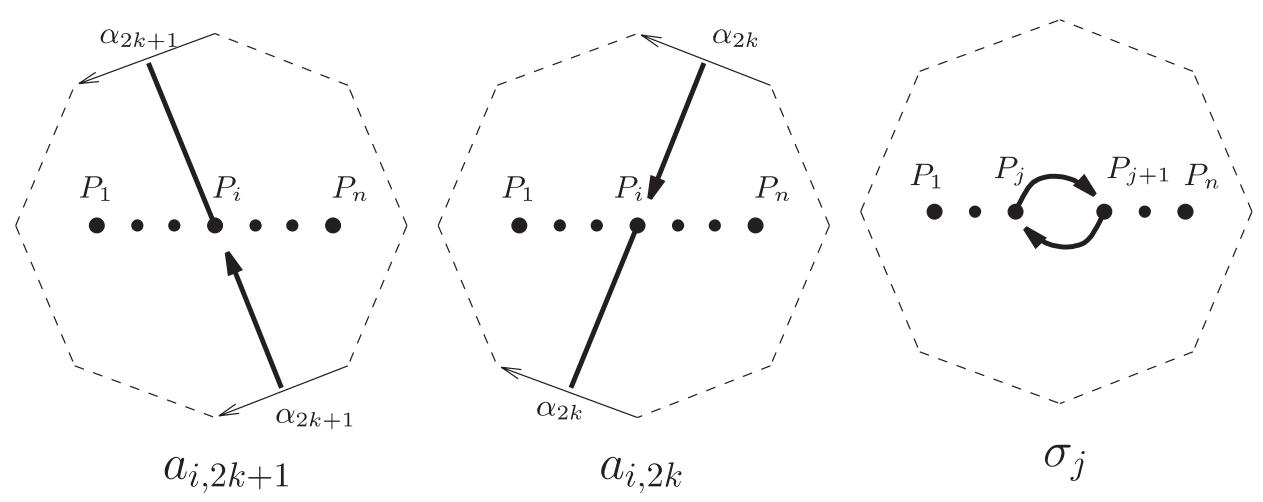

FiguRE 4. Generators for $B_{n}(M)$.

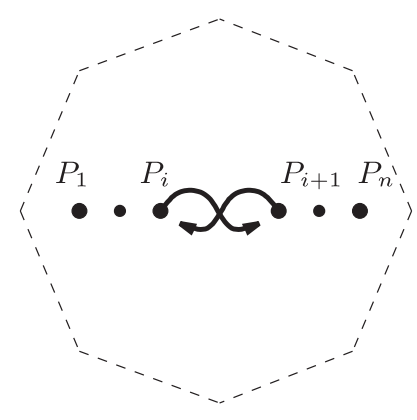

$\tau_{i}$

Figure 5. The singular braid $\tau_{i}$.

For every $i=1, \ldots, n-1$, we define the singular braid $\tau_{i} \in S_{1} B_{n}(M)$ as in Figure 5. This singular braid has a unique singular point, which is the intersection of the $i$-th string and the $(i+1)$-th string. The $i$-th string goes from $\left(P_{i}, 0\right)$ to $\left(P_{i+1}, 1\right)$, and the $(i+1)$-th string goes from $\left(P_{i+1}, 0\right)$ to $\left(P_{i}, 1\right)$. The other strings are trivial. 
By a suitable isotopy, any singular braid $b \in S_{k} B_{n}(M)$ can be written in the form

$$
b=c_{1} \tau_{j_{1}} c_{2} \tau_{j_{2}} \cdots c_{k} \tau_{j_{k}} c_{k+1}
$$

where $c_{i} \in B_{n}(M)$. So the following set generates $S B_{n}(M)$ (as a monoid):

$$
\left\{a_{i, r}^{ \pm 1} ; i=1, \ldots, n, r=1, \ldots, 2 g\right\} \cup\left\{\sigma_{1}^{ \pm 1}, \ldots, \sigma_{n-1}^{ \pm 1}\right\} \cup\left\{\tau_{1}, \ldots, \tau_{n-1}\right\} .
$$

Now, the morphism $\eta: S B_{n}(M) \rightarrow \mathbb{Z}\left[B_{n}(M)\right]$ sends $\sigma_{i}^{ \pm 1}$ to $\sigma_{i}^{ \pm 1}, a_{i, r}^{ \pm 1}$ to $a_{i, r}^{ \pm 1}$ and $\tau_{i}$ to $\sigma_{i}-\sigma_{i}^{-1}$. Recall that $V_{d}$ denotes the $\mathbb{Z}$-submodule of $\mathbb{Z}\left[B_{n}(M)\right]$ generated by $\eta\left(S_{d} B_{n}(M)\right)$. From the above considerations, we immediately get

Proposition 2.1. Let $J$ be the two-sided ideal of $\mathbb{Z}\left[B_{n}(M)\right]$ generated by $\left\{\sigma_{i}-\right.$ $\left.\sigma_{i}^{-1} ; i=1, \ldots, n-1\right\}$. Then $V_{d}=J^{d}$ for all $d \in \mathbb{N}$.

2.2. From $J^{d}$ to $I\left(K_{n}\right)^{d}$. Recall that $H_{n}$ denotes the semi-direct product $\pi_{1}(M)^{n}$ $\rtimes \Sigma_{n}$. We define a homomorphism $\varphi: B_{n}(M) \rightarrow H_{n}$ as follows. We fix a disc $D$ embedded in $M$ which contains $\mathcal{P}$ and, for all $i, j \in\{1, \ldots, n\}$, a path $\alpha_{i, j}$ in $D$ going from $P_{i}$ to $P_{j}$. Pick a braid $b=\left(b_{1}, \ldots, b_{n}\right), b_{i}:[0,1] \rightarrow M \times[0,1]$, based at $\mathcal{P}$. Let $s \in \Sigma_{n}$ be the permutation induced by $b$. Let $\bar{b}_{i}:[0,1] \rightarrow M$ be the projection of $b_{i}$ on the first coordinate, and let $\mu_{i}$ be the loop based at $P_{i}$ defined by $\mu_{i}=\bar{b}_{i} \alpha_{s(i), i}$. Then we set

$$
\varphi(b)=\left(\mu_{1}, \ldots, \mu_{n}\right) s \in \pi_{1}(M)^{n} \rtimes \Sigma_{n}=H_{n} .
$$

One can easily verify that $\varphi: B_{n}(M) \rightarrow H_{n}$ is a well-defined homomorphism, and that its definition depends on the choice of $D$ but not on the choice of the paths $\alpha_{i, j}$.

Let $K_{n}$ denote the kernel of $\varphi$. It is a classical matter that a set-section $\sigma$ : $H_{n} \rightarrow B_{n}(M)$ of $\varphi$ determines a $\mathbb{Z}$-isomorphism $\Phi: \mathbb{Z}\left[B_{n}(M)\right] \rightarrow \mathbb{Z}\left[K_{n}\right] \otimes \mathbb{Z}\left[H_{n}\right]$ defined by

$$
\Phi(b)=b(\sigma \circ \varphi)(b)^{-1} \otimes \varphi(b) .
$$

Let us fix such a set-section.

Recall that the augmentation ideal of a group $G$ is defined to be the two-sided ideal $I(G)$ of $\mathbb{Z}[G]$ generated by the set $\{1-g ; g \in G\}$. In this subsection, we prove the following.

Proposition 2.2. The isomorphism $\Phi: \mathbb{Z}\left[B_{n}(M)\right] \rightarrow \mathbb{Z}\left[K_{n}\right] \otimes \mathbb{Z}\left[H_{n}\right]$ sends $J^{d}$ isomorphically to $I\left(K_{n}\right)^{d} \otimes \mathbb{Z}\left[H_{n}\right]$ for all $d \in \mathbb{N}$.

Note that Proposition 2.2 implies that, in order to prove Theorem 1.2, it will suffice to prove the following two conditions:

(1) $\bigcap_{d=0}^{\infty} I\left(K_{n}\right)^{d}=\{0\}$, and

(2) $I\left(K_{n}\right)^{d} / I\left(K_{n}\right)^{d+1}$ is a free $\mathbb{Z}$-module for all $d \geq 0$.

To prove Proposition 2.2 we will make use of some classical exact sequences involving braid groups (see [5]). The first one comes from the homomorphism $\pi$ which maps a given braid to the permutation that it induces on $\mathcal{P}$. The kernel of this (clearly well-defined) homomorphism is a subgroup of $B_{n}(M)$ denoted by $P B_{n}(M)$, whose elements are called pure braids. Then one has

$$
1 \longrightarrow P B_{n}(M) \longrightarrow B_{n}(M) \stackrel{\pi}{\longrightarrow} \Sigma_{n} \longrightarrow 1 \text {. }
$$


On the other hand, there is a homomorphism $\varrho: P B_{n}(M) \rightarrow P B_{n-1}(M)$ which sends $\left(b_{1}, \ldots, b_{n}\right)$ to $\left(b_{2}, \ldots, b_{n}\right)$. If we set $\mathcal{P}_{n-1}=\left\{P_{2}, \ldots, P_{n}\right\}$, then the kernel of $\varrho$ can be seen as the group $\pi_{1}\left(M \backslash \mathcal{P}_{n-1}\right)$. This gives

$$
1 \longrightarrow \pi_{1}\left(M \backslash \mathcal{P}_{n-1}\right) \longrightarrow P B_{n}(M) \stackrel{\varrho}{\longrightarrow} P B_{n-1}(M) \longrightarrow 1
$$

Finally, if $b$ is a pure braid, the projection of each string $b_{i}(i \in\{1, \ldots, n\})$ over $M$, denoted by $\overline{b_{i}}$, is a loop in $M$ based at $P_{i}$, which determines an element $\mu_{i} \in \pi_{1}(M)$. This gives a homomorphism $\theta: P B_{n}(M) \rightarrow \pi_{1}(M)^{n}$, which sends $\left(b_{1}, \ldots, b_{n}\right)$ to $\left(\mu_{1}, \ldots, \mu_{n}\right)$. One can easily verify that $K_{n}=\operatorname{ker} \theta$, and that the exact sequence

$$
1 \longrightarrow K_{n} \longrightarrow P B_{n}(M) \stackrel{\theta}{\longrightarrow} \pi_{1}(M)^{n} \longrightarrow 1
$$

extends to the exact sequence

$$
1 \longrightarrow K_{n} \longrightarrow B_{n}(M) \stackrel{\varphi}{\longrightarrow} H_{n} \longrightarrow 1 \text {. }
$$

Moreover, $K_{n}$ is the normal closure in $P B_{n}(M)$ of the subgroup $P B_{n}(D)$, where $D$ is a disc in $M$ which contains $\mathcal{P}$ (see [5]).

In what follows, we write $I=I\left(K_{n}\right)$ and we consider $\mathbb{Z}\left[K_{n}\right]$ as a subring of $\mathbb{Z}\left[B_{n}(M)\right]$. The next lemma is a preliminary to the proof of Proposition 2.2 .

Lemma 2.3. Let $B=\mathbb{Z}\left[B_{n}(M)\right]$. For every $d \geq 1$, one has

$$
J^{d}=B I^{d} B=B I^{d}=I^{d} B \text {. }
$$

Proof. Since $K_{n}$ is a normal subgroup of $B_{n}(M)$, it is straightforward to prove that $B I^{d} B=B I^{d}=I^{d} B$. So, it suffices to prove that $J=B I B$.

The inclusion $J \subset B I B$ is obvious, once we notice that $\sigma_{i}^{2} \in K_{n}$ and that $\sigma_{i}-\sigma_{i}^{-1}=\sigma_{i}^{-1}\left(\sigma_{i}^{2}-1\right) \in B I B$. For the other inclusion, we must prove that for all $p \in K_{n}$ one has $p-1 \in J$. Suppose that $p=p_{1} p_{2}$, with $p_{1}, p_{2} \in K_{n}$; then $p-1=p_{1}\left(p_{2}-1\right)+\left(p_{1}-1\right)$, so it suffices to show it for a set of generators of $K_{n}$. As we said before, $K_{n}$ is the normal closure of $P B_{n}(D)$ in $P B_{n}(M)$, so a set of generators of $K_{n}$ consists of elements of the form $\alpha b \alpha^{-1}$, where $\alpha \in P B_{n}(M)$ and $b \in P B_{n}(D)$.

Take an element $\alpha b \alpha^{-1}$ as above. One has: $\alpha b \alpha^{-1}-1=\alpha(b-1) \alpha^{-1}$, so we only have to show that $b-1 \in J$ for $b \in P B_{n}(D)$. It is known ([16], Lemma 1.2) that $b-1$ belongs to the ideal of $\mathbb{Z}\left[B_{n}(D)\right]$ generated by $\left\{\sigma_{i}-\sigma_{i}^{-1} ; i=1, \ldots, n-1\right\}$. But the extension of this ideal to $\mathbb{Z}\left[B_{n}(M)\right] \supset \mathbb{Z}\left[B_{n}(D)\right]$ is precisely $J$, so $b-1 \in J$.

Proof of Proposition [2.2. First, we show that $\Phi\left(J^{d}\right) \subset I^{d} \otimes \mathbb{Z}\left[H_{n}\right]$ for all $d \geq 1$. By Lemma 2.3, we know that $J^{d}=I^{d} B$; thus $J^{d}$ is generated as a $\mathbb{Z}$-module by the elements of the form $\left(k_{1}-1\right) \cdots\left(k_{d}-1\right) b$, where $b \in B_{n}(M)$ and $k_{i} \in K_{n}$ for $i=1, \ldots, d$. Now, the image of such an element by $\Phi$ is $\left(k_{1}-1\right) \cdots\left(k_{d}-1\right) b^{\prime} \otimes \varphi(b)$, where $b^{\prime}=b(\sigma \circ \varphi)(b)^{-1}$, which clearly belongs to $I^{d} \otimes \mathbb{Z}\left[H_{n}\right]$.

The inclusion $I^{d} \otimes \mathbb{Z}\left[H_{n}\right] \subset \Phi\left(J^{d}\right)$ follows from the facts that $I^{d} \otimes \mathbb{Z}\left[H_{n}\right]$ is generated as a $\mathbb{Z}$-module by the elements of the form $\left(k_{1}-1\right) \cdots\left(k_{d}-1\right) k \otimes \beta$, where $k_{1}, \ldots, k_{d}, k \in K_{n}$ and $\beta \in H_{n}$, and that such an element is the image by $\Phi$ of $\left(k_{1}-1\right) \cdots\left(k_{d}-1\right) k \sigma(\beta) \in I^{d} B=J^{d}$.

2.3. The structure of $K_{n}$. The goal of this subsection is to prove the following.

Proposition 2.4. For $n \geq 2$, there exists a free group $F_{n}$ such that $K_{n}=F_{n} \rtimes$ $K_{n-1}$. Moreover, the action of $K_{n-1}$ on the abelianization of $F_{n}$ is trivial. 
Remarks. $\quad$ (i) The notation $F_{n}$ may lead to some confusion; indeed, here $F_{n}$ is not a free group of rank $n$. It is actually of infinite rank.

(ii) A direct consequence of Proposition 2.4 is that $K_{n}$ can be expressed as an iterated semi-direct product of (infinitely generated) free groups

$$
K_{n}=F_{n} \rtimes\left(F_{n-1} \rtimes\left(\cdots \rtimes\left(F_{3} \rtimes F_{2}\right) \cdots\right)\right) .
$$

Recall the exact sequences defined in the previous subsection. Since $K_{n}$ is a subgroup of $P B_{n}(M)$, we can consider the image by $\varrho$ of $K_{n}$. By definition, it is equal to $K_{n-1}$. If we denote $F_{n}=\operatorname{ker} \varrho \cap K_{n}$, we obtain the following commutative diagram, where all rows and columns are exact:

$$
\begin{array}{ccccccc} 
& 1 & & 1 & & 1 & \\
& \uparrow & & \uparrow & & \uparrow & \\
1 \rightarrow & \pi_{1}\left(M, P_{1}\right) & \rightarrow & \pi_{1}(M)^{n} & \rightarrow & \pi_{1}(M)^{n-1} & \rightarrow 1 \\
& \uparrow & & \uparrow \theta & & \uparrow \theta & \\
1 \rightarrow & \pi_{1}\left(M \backslash \mathcal{P}_{n-1}\right) & \rightarrow & P B_{n}(M) & \stackrel{\varrho}{\rightarrow} & P B_{n-1}(M) & \rightarrow 1 \\
& \uparrow & & \uparrow & & \uparrow & \\
1 \rightarrow & F_{n} & \rightarrow & K_{n} & \stackrel{\varrho}{\rightarrow} & K_{n-1} & \rightarrow 1 \\
& \uparrow & & \uparrow & & \uparrow & \\
& 1 & & 1 & & 1 &
\end{array}
$$

Notice that $F_{n}$ is a free group, since it is a subgroup of $\pi_{1}\left(M \backslash \mathcal{P}_{n-1}\right)$, which is a free group. We are especially interested in the lowest row of the diagram. In particular, in order to show Proposition 2.4, we will show that there exists a homomorphism $s: K_{n-1} \rightarrow K_{n}$ which is a section of $\varrho$, and that $K_{n-1}$ acts trivially on the abelianization of $F_{n}$. We turn first to find a free set of generators for $F_{n}$.

Let $\Omega=\left\{\omega_{1}, \ldots, \omega_{2 g}\right\}$ be a set of $2 g$ letters. It is well known that a presentation for $\pi_{1}(M)$ is as follows:

$$
\pi_{1}(M)=\left\langle\Omega ; \quad\left(\omega_{1} \omega_{2} \cdots \omega_{2 g} \omega_{1}^{-1} \omega_{2}^{-1} \cdots \omega_{2 g}^{-1}\right)=1\right\rangle .
$$

For every element $\gamma \in \pi_{1}(M)$ we choose a unique word $\widetilde{\gamma}$ over $\Omega \cup \Omega^{-1}$ which represents $\gamma$. We call this word the normal form of $\gamma$. Normal forms are chosen in such a way that they are prefix-closed (namely, if $\omega_{1} \omega_{2}$ is a normal form, then $\omega_{1}$ is also a normal form). For every word $\omega$ over $\Omega \cup \Omega^{-1}$, we will denote by $\omega_{(i)}$ the word over $\left\{a_{i, 1}^{ \pm 1}, \ldots, a_{i, 2 g}^{ \pm 1}\right\}$ obtained from $\omega$ by replacing $\omega_{j}^{ \pm 1}$ by $a_{i, j}^{ \pm 1}$, for all $j=1, \ldots, 2 g$.

Let us consider, for $1 \leq i<j \leq n$, the braid $T_{i, j}$ drawn in Figure 6. All its strings are trivial except the $i$-th one, which goes around the points $P_{i+1}, \ldots, P_{j}$ and turns back to $P_{i}$.

Notice that in $\pi_{1}\left(M \backslash \mathcal{P}_{n-1}\right)$, viewed as a subgroup of $P B_{n}(M)$, one has

$$
T_{1, n}=a_{1,1} \cdots a_{1,2 g} a_{1,1}^{-1} \cdots a_{1,2 g}^{-1} .
$$

Lemma 2.5. The following set is a free system of generators for $F_{n}$.

$$
\mathcal{B}=\left\{\widetilde{\gamma}_{(1)} T_{1, j} \widetilde{\gamma}_{(1)}^{-1} ; 2 \leq j \leq n \text { and } \gamma \in \pi_{1}(M)\right\} .
$$

Proof. Consider the Cayley graph of $\pi_{1}(M)$, which is defined as follows. Its vertices are the elements of $\pi_{1}(M)$, and its edges are labeled by $\Omega$. For every vertex $\gamma \in$ $\pi_{1}(M)$ and for every $i \in 1, \ldots, 2 g$, there is exactly one edge labeled by $\omega_{i}$, with source $\gamma$ and target $\gamma \omega_{i}$. 


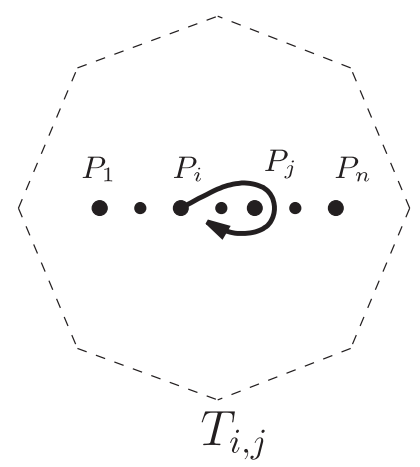

FIgURE 6 . The path (or braid) $T_{i, j}$.

In this graph, the normal form of an element $\gamma \in \pi_{1}(M)$ corresponds to a unique path going from 1 to $\gamma$. By the prefix-closed condition mentioned above, the set of normal forms of $\pi_{1}(M)$ defines a maximal tree $T$ of the Cayley graph.

The Cayley graph of $\pi_{1}(M)$ can be seen as the one-skeleton of a tiling of the (euclidean or hyperbolic) plane. For every vertex $\gamma$, the path which starts at $\gamma$ and which is labeled by $\omega_{1} \ldots \omega_{2 g} \omega_{1}^{-1} \ldots \omega_{2 g}^{-1}$ bounds a fundamental region $R_{\gamma}$ of this tiling, and all fundamental regions are obtained in this way. Hence, there is a one-to-one correspondence between the vertices of the Cayley graph and its fundamental regions. Therefore, the fundamental group of the Cayley graph of $\pi_{1}(M)$ is the free group with free system of generators

$$
\left\{\widetilde{\gamma}\left(\omega_{1} \ldots \omega_{2 g} \omega_{1}^{-1} \ldots \omega_{2 g}^{-1}\right) \widetilde{\gamma}^{-1} ; \gamma \in \pi_{1}\left(M, P_{1}\right)\right\} .
$$

We now define a graph $\Gamma$ as follows. Take the Cayley graph of $\pi_{1}(M)$ and replace the labels $\omega_{i}$ by $a_{1, i}$. Then, for every vertex $\gamma$, add $n-2$ edges with source and target $\gamma$, labeled by $T_{1,2}, \ldots, T_{1, n-1}$, respectively. Notice that the fundamental group of $\Gamma$ is the free group with free system of generators $\mathcal{B}$, where $T_{1, n}=a_{1,1} \cdots a_{1,2 g} a_{1,1}^{-1} \cdots a_{1,2 g}^{-1}$.

Recall the exact sequence

$$
1 \longrightarrow F_{n} \longrightarrow \pi_{1}\left(M \backslash \mathcal{P}_{n-1}\right) \stackrel{\theta}{\longrightarrow} \pi_{1}\left(M, P_{1}\right) \longrightarrow 1 .
$$

One can easily verify that $\pi_{1}\left(M \backslash \mathcal{P}_{n-1}\right)$ is freely generated by $\left\{a_{1,1}, \ldots, a_{1,2 g}\right.$, $\left.T_{1,2}, \ldots, T_{1, n-1}\right\}$, and that $\theta$ sends $a_{1, i}$ to $\omega_{i}$ for all $i=1, \ldots, 2 g$, and sends $T_{1, j}$ to 1 for all $j=2, \ldots, n-1$. It follows from classical geometric methods (see [11, Chapter III, Proposition 3.2) that the group $F_{n}$ is the fundamental group of the graph $\Gamma$, hence $\mathcal{B}$ is a free system of generators for $F_{n}$, as we wanted to prove.

Lemma 2.6. There is a homomorphism $\sigma: K_{n-1} \rightarrow K_{n}$ which is a section of $\varrho: K_{n} \rightarrow K_{n-1}$.

Proof. The case $n=2$ is trivial, since $K_{1}=\operatorname{ker}\left(\pi_{1}(M) \stackrel{\theta}{\rightarrow} \pi_{1}(M)\right)=1$. Hence $K_{2}=F_{2}$ is a free group of infinite rank. Suppose now that $n>2$. The image of a given braid by $\sigma$ is obtained by doubling the first string (the one starting at $P_{2}$ ). This can be done for elements of $K_{n}$ since we can first isotope this string to a straight line, and then double it, as follows. 
It is well known that the kernel of the homomorphism $\theta_{2}: P B_{n}(M) \rightarrow \pi_{1}\left(M, P_{2}\right)$ is $P B_{n-1}\left(M \backslash\left\{P_{2}\right\}\right)$ (see [5]). Moreover, one can easily see that $K_{n}$ lies in this kernel, namely $K_{n} \subset P B_{n-1}\left(M \backslash\left\{P_{2}\right\}\right)$. Similarly, one has $K_{n-1} \subset P B_{n-2}\left(M \backslash\left\{P_{2}\right\}\right)$. The homomorphism $\varrho_{n}: P B_{n-1}\left(M \backslash\left\{P_{2}\right\}\right) \longrightarrow P B_{n-2}\left(M \backslash\left\{P_{2}\right\}\right)$ which sends $\left(b_{1}, b_{3}, \ldots, b_{n}\right)$ to $\left(b_{3}, \ldots, b_{n}\right)$ is the restriction of $\varrho$ to $P B_{n-1}\left(M \backslash\left\{P_{2}\right\}\right)$. In particular, it sends $K_{n}$ onto $K_{n-1}$.

We consider an embedding $f: M \backslash\left\{P_{2}\right\} \rightarrow M \backslash\left\{P_{2}\right\}$ satisfying:

- $f\left(P_{i}\right)=P_{i}$ for $i=3, \ldots, n$;

- $P_{1}$ does not lie in the image of $f$;

- $f$ is homotopy equivalent (relative to $\left\{P_{3}, \ldots, P_{n}\right\}$ ) to the identity.

Then, $f$ induces a homomorphism $\sigma: P B_{n-2}\left(M \backslash\left\{P_{2}\right\}\right) \rightarrow P B_{n-1}\left(M \backslash\left\{P_{2}\right\}\right)$, which sends $\left(b_{3}, \ldots, b_{n}\right)$ to $\left(1_{P_{1}},(f \times \mathrm{id}) b_{3}, \ldots(f \times \mathrm{id}) b_{n}\right)$. By the third condition, this homomorphism is a section of $\varrho_{n}$. It obviously sends $K_{n-1}$ to $K_{n}$.

Now, $K_{n-1}$ acts on $F_{n}$ in the following way: Given $b \in K_{n-1}$, the action induced by $b$ sends $f \in F_{n}$ to $\sigma(b) f \sigma(b)^{-1}$. This action induces an action of $K_{n-1}$ on the abelianization $F_{n} /\left[F_{n}, F_{n}\right]$ of $F_{n}$ (here $\left[F_{n}, F_{n}\right]$ denotes the commutator subgroup of $F_{n}$ ). The proof of Proposition 2.4 is finally obtained from the following result.

Lemma 2.7. The action of $K_{n-1}$ on the abelianization of $F_{n}$ is trivial.

Proof. We only need to verify that the action of the generators of $K_{n-1}$ on the generators of $F_{n}$ is trivial after abelianization. Moreover, let us see that it suffices to show the result for the action defined by any set-map section $s$ of $\varrho$.

Indeed, if $s$ is a section of $\varrho$, then for every $b \in K_{n-1}$, there exists an element $\widehat{b} \in F_{n}$ such that $\sigma(b)=\widehat{b} s(b)$. Therefore, if $K_{n-1}$ acts trivially on $F_{n} /\left[F_{n}, F_{n}\right]$ via $s$, we obtain, for every $f \in F_{n}$,

$$
\sigma(b) f \sigma(b)^{-1} \equiv \widehat{b}\left(s(b) f s(b)^{-1}\right) \widehat{b}^{-1} \equiv \widehat{b} f \widehat{b}^{-1} \equiv f \quad\left(\bmod \left[F_{n}, F_{n}\right]\right) .
$$

As we said in Subsection 2.2. a set of generators for $K_{n-1}$ consists of elements of the form $\alpha b \alpha^{-1}$, where $\alpha \in P B_{n-1}(M)$ and $b \in P B_{n-1}(D)$. On the other hand, it is known that $T=\left\{T_{i, j} \mid \quad 2 \leq i<j \leq n\right\}$ is a set of generators for $P B_{n-1}(D)$, where $T_{i, j}$ denotes the braid defined in Subsection [2.1. Therefore, the following is a set of generators for $K_{n-1}$ :

$$
\left\{\alpha T_{i, j} \alpha^{-1} ; 2 \leq i<j \leq n \text {, and } \alpha \text { is a word over }\left\{a_{k, r}^{ \pm 1} ; 2 \leq k \leq n, 1 \leq r \leq 2 g\right\}\right\} \text {. }
$$

We take $s$ such that $s\left(\alpha T_{i, j} \alpha^{-1}\right)=\alpha T_{i, j} \alpha^{-1} \in K_{n}$. In other words, we just add a trivial string based at $P_{1}$ for any element of the set of generators of $K_{n-1}$. We remark that $s$ is a set map section of $\varrho$, but it is not a homomorphism.

Now, $F_{n}$ is by definition a normal subgroup of $P B_{n}(M)$. Therefore, if we show that each $T_{i, j} \quad(i \geq 2)$ acts trivially on $F_{n} /\left[F_{n}, F_{n}\right]$ by conjugation, then we will have finished the proof, since in that case

$$
\begin{gathered}
\left(\alpha T_{i, j} \alpha^{-1}\right) f\left(\alpha T_{i, j}^{-1} \alpha^{-1}\right) \equiv \alpha T_{i, j}\left(\alpha^{-1} f \alpha\right) T_{i, j}^{-1} \alpha^{-1} \\
\equiv \alpha\left(\alpha^{-1} f \alpha\right) \alpha^{-1} \equiv f \quad\left(\bmod \left[F_{n}, F_{n}\right]\right) .
\end{gathered}
$$

Recall from Lemma 2.5 that

$$
\mathcal{B}=\left\{\widetilde{\gamma}_{(1)} T_{1, k} \widetilde{\gamma}_{(1)}^{-1} ; 2 \leq k \leq n \text { and } \gamma \in \pi_{1}(M)\right\}
$$


is a free system of generators for $F_{n}$, where $\widetilde{\gamma}_{(1)}$ is a word over $\left\{a_{1,1}^{ \pm 1}, \ldots, a_{1,2 g}^{ \pm 1}\right\}$ for all $\gamma \in \pi_{1}(M)$. One can verify (just drawing the corresponding braids) that in $P B_{n}(M)$ one has the following relations:

$T_{i, j} a_{1, r} T_{i, j}^{-1}=a_{1, r}$

$T_{i, j} T_{1, k} T_{i, j}^{-1}=T_{1, k} \quad \quad(k<i$ or $k \geq j)$,

$T_{i, j} T_{1, k} T_{i, j}^{-1}=T_{1, i-1} T_{1, i}^{-1} T_{1, k} T_{1, j}^{-1} T_{1, i} T_{1, i-1}^{-1} T_{1, j} \equiv T_{1, k} \quad\left(\bmod \left[F_{n}, F_{n}\right]\right)(i \leq k<j)$, where $2 \leq i<j \leq n, r \in\{1, \ldots, 2 g\}$ and $k \in\{2, \ldots, n\}$.

Therefore, in $F_{n} /\left[F_{n}, F_{n}\right]$, we have

$T_{i, j}\left(\widetilde{\gamma}_{(1)} T_{1, k} \widetilde{\gamma}_{(1)}^{-1}\right) T_{i, j}^{-1}=\widetilde{\gamma}_{(1)}\left(T_{i, j} T_{1, k} T_{i, j}^{-1}\right) \widetilde{\gamma}_{(1)}^{-1} \equiv \widetilde{\gamma}_{(1)} T_{1, k} \widetilde{\gamma}_{(1)}^{-1} \quad\left(\bmod \left[F_{n}, F_{n}\right]\right)$, as we wanted to show.

2.4. Proof of Theorem 1.2. Let $A$ and $C$ be two groups such that $C$ acts on $A$. For $a \in A$ and $c \in C$, we denote by $a^{c}$ the action of $c$ on $a$. Then, the $\mathbb{Z}$-module $\mathbb{Z}[A] \otimes \mathbb{Z}[C]$ carries a natural structure of $\mathbb{Z}$-algebra, where the multiplication is defined by

$$
\left(a_{1} \otimes c_{1}\right) \cdot\left(a_{2} \otimes c_{2}\right)=\left(a_{1} a_{2}^{c_{1}}\right) \otimes\left(c_{1} c_{2}\right) .
$$

Moreover, this algebra is naturally isomorphic to $\mathbb{Z}[A \rtimes C]$ via an isomorphism which sends $a \otimes c$ to $a c$ for all $a \in A$ and all $c \in C$.

Recall that the augmentation ideal of a group $G$ is denoted by $I(G)$. The following lemma will be used to prove Theorem 1.2. Its proof can be found in 13 . Lemma 3.1].

Lemma 2.8. Let $A$ and $C$ be two groups. Assume that an action of $C$ on $A$ is given, and that this action induces the trivial action on the abelianization of $A$. Then one has

$$
I(A \rtimes C)^{m}=\sum_{k=0}^{m} I(A)^{k} \otimes I(C)^{m-k}
$$

for all $m \geq 0$.

Proof of Theorem 1.2. As pointed out in Subsection 2.2, it suffices to prove the following two conditions.

(1) $\bigcap_{d=0}^{\infty} I\left(K_{n}\right)^{d}=\{0\}$, and

(2) $I\left(K_{n}\right)^{d} / I\left(K_{n}\right)^{d+1}$ is a free $\mathbb{Z}$-module for all $d \geq 0$.

We argue by induction on $n$. The case $n=1$ is trivial, since $I\left(K_{1}\right)=I(\{1\})=0$. So, we assume that $n \geq 2$ and that conditions 1 and 2 hold for $K_{p}$, where $p<n$.

The group $F_{n}$ is free; thus, by [8], one has

(1) $\bigcap_{d=0}^{\infty} I\left(F_{n}\right)^{d}=\{0\}$, and

(2) $I\left(F_{n}\right)^{d} / I\left(F_{n}\right)^{d+1}$ is a free $\mathbb{Z}$-module for all $d \geq 0$.

These two properties imply that $I\left(F_{n}\right)^{d}$ is a free $\mathbb{Z}$-module and that

$$
I\left(F_{n}\right)^{d} \simeq \bigoplus_{k=d}^{\infty} I\left(F_{n}\right)^{k} / I\left(F_{n}\right)^{k+1}
$$

for all $d \geq 0$. We choose a $\mathbb{Z}$-basis $\mathcal{B}_{d}$ of $I\left(F_{n}\right)^{d} / I\left(F_{n}\right)^{d+1}$ for all $d \geq 0$. From the above isomorphism one has that $\mathcal{B}_{\geq d}=\coprod_{k=d}^{\infty} \mathcal{B}_{k}$ is a $\mathbb{Z}$-basis of $I\left(F_{n}\right)^{d}$.

From the induction hypothesis, one also has

(1) $\bigcap_{d=0}^{\infty} I\left(K_{n-1}\right)^{d}=\{0\}$, and

(2) $I\left(K_{n-1}\right)^{d} / I\left(K_{n-1}\right)^{d+1}$ is a free $\mathbb{Z}$-module for all $d \geq 0$. 
This implies that $I\left(K_{n-1}\right)^{d}$ is a free $\mathbb{Z}$-module and that

$$
I\left(K_{n-1}\right)^{d} \simeq \bigoplus_{k=d}^{\infty} I\left(K_{n-1}\right)^{k} / I\left(K_{n-1}\right)^{k+1}
$$

for all $d \geq 0$. We choose a $\mathbb{Z}$-basis $\mathcal{C}_{d}$ of $I\left(K_{n-1}\right)^{d} / I\left(K_{n-1}\right)^{d+1}$ for all $d \geq 0$. Thus $\mathcal{C}_{\geq d}=\coprod_{k=d}^{\infty} \mathcal{C}_{k}$ is a $\mathbb{Z}$-basis of $I\left(K_{n-1}\right)^{d}$.

Now, by Proposition 2.4 $F_{n}$ and $K_{n-1}$ satisfy the hypothesis of Lemma 2.8 Hence, we have the equality

$$
I\left(K_{n}\right)^{m}=\sum_{d=0}^{m} I\left(F_{n}\right)^{d} \otimes I\left(K_{n-1}\right)^{m-d}
$$

for all $m \geq 0$. From this equality, one can easily verify that the set

$$
\mathcal{D}_{\geq m}=\left\{b \otimes c \in \mathbb{Z}\left[F_{n}\right] \otimes \mathbb{Z}\left[K_{n-1}\right] ; \quad b \in \mathcal{B}_{i}, c \in \mathcal{C}_{j}, i+j \geq m\right\}
$$

is a generating set for $I\left(K_{n}\right)^{m}$. Since this set is linearly independent, $I\left(K_{n}\right)^{m}$ is a free $\mathbb{Z}$-module whose basis is $\mathcal{D}_{\geq m}$. It follows that $I\left(K_{n}\right)^{m} / I\left(K_{n}\right)^{m+1}$ is a free $\mathbb{Z}$-module with basis

$$
\mathcal{D}_{m}=\mathcal{D}_{\geq m} \backslash \mathcal{D}_{\geq m+1}=\left\{b \otimes c \in \mathbb{Z}\left[F_{n}\right] \otimes \mathbb{Z}\left[K_{n-1}\right] ; \quad b \in \mathcal{B}_{i}, c \in \mathcal{C}_{j}, i+j=m\right\},
$$

and that $\bigcap_{d=0}^{\infty} I\left(K_{n}\right)^{d}=\{0\}$, since $\mathcal{D}_{\geq 0}$ is a basis for $\mathbb{Z}\left[K_{n}\right]$.

\section{The universal VASSILIEV INVARIANT}

The proof of Theorem 1.3 is divided into five steps. In what follows each subsection will correspond to one of them.

The first subsection is dedicated to the definition of a linear map

$$
u: \mathbb{Z}\left[B_{n}(M)\right] \longrightarrow \widehat{\mathcal{A}}_{n} \rtimes \mathbb{Z}\left[H_{n}\right] .
$$

Recall that $\mathbb{Z}\left[B_{n}(M)\right]$ is isomorphic to $\mathbb{Z}\left[K_{n}\right] \otimes \mathbb{Z}\left[H_{n}\right]$ as a $\mathbb{Z}$-module (see Subsection $[2.2)$, and notice that $\widehat{\mathcal{A}}_{n} \rtimes \mathbb{Z}\left[H_{n}\right]$ is equal as a $\mathbb{Z}$-module to $\widehat{\mathcal{A}}_{n} \otimes \mathbb{Z}\left[H_{n}\right]$. Hence, we will only need to define a linear map $v: \mathbb{Z}\left[K_{n}\right] \rightarrow \widehat{\mathcal{A}}_{n}$.

Recall that the subgroups $G_{i}$ of the lower central series of a group $G$ are defined recursively by $G_{1}=G$ and $G_{i+1}=\left[G, G_{i}\right]$ for $i \geq 1$. The associated graded Lie algebra of $G$ is defined by $\operatorname{gr}(G)=\bigoplus_{i>1} G_{i} / G_{i+1}$. It is a graded Lie algebra over $\mathbb{Z}$ whose enveloping algebra is denoted by $\mathcal{U} \operatorname{gr}(G)$. In Subsection 3.2 we construct a homomorphism $\chi_{1}: \mathcal{A}_{n} \rightarrow \mathcal{U} \operatorname{gr}\left(K_{n}\right)$ of $\mathbb{Z}$-algebras, and we prove that this homomorphism is actually an isomorphism.

Write $I=I\left(K_{n}\right)$. In Subsection 3.3 we construct an isomorphism of $\mathbb{Z}$-algebras $\chi_{2}: \mathcal{U} \operatorname{gr}\left(K_{n}\right) \rightarrow \operatorname{gr}_{I} \mathbb{Z}\left[K_{n}\right]$, using a result due to Quillen.

In Subsection 3.4 we consider the isomorphism $\chi=\chi_{2} \circ \chi_{1}: \mathcal{A}_{n} \rightarrow \operatorname{gr}_{I} \mathbb{Z}\left[K_{n}\right]$ and we prove that $\operatorname{gr} v$ is the inverse of $\chi$. It will immediately follow that grv: $\operatorname{gr}_{I} \mathbb{Z}\left[K_{n}\right] \rightarrow \mathcal{A}_{n}$ is an isomorphism of $\mathbb{Z}$-algebras, and that gru: $\operatorname{gr}_{V} \mathbb{Z}\left[B_{n}(M)\right] \rightarrow$ $\mathcal{A}_{n} \rtimes \mathbb{Z}\left[H_{n}\right]$ is an isomorphism of $\mathbb{Z}$-modules.

Finally, we prove in Subsection 3.5 that gru is a homomorphism of $\mathbb{Z}$-algebras. This will finish the proof of Theorem[1.3. 
3.1. Construction of $u: \mathbb{Z}\left[B_{n}(M)\right] \longrightarrow \widehat{\mathcal{A}}_{n} \rtimes \mathbb{Z}\left[H_{n}\right]$. From now on we fix a setsection $\sigma: H_{n} \rightarrow B_{n}(M)$ of $\varphi: B_{n}(M) \rightarrow H_{n}$. As we pointed out in Subsection 2.2 this set-section leads to an isomorphism $\Phi: \mathbb{Z}\left[B_{n}(M)\right] \longrightarrow \mathbb{Z}\left[K_{n}\right] \otimes \mathbb{Z}\left[H_{n}\right]$ of $\mathbb{Z}$ modules.

We turn now to define a linear homomorphism $v: \mathbb{Z}\left[K_{n}\right] \rightarrow \widehat{\mathcal{A}}_{n}$. Then we will set

$$
u=(v \otimes \mathrm{id}) \circ \Phi: \mathbb{Z}\left[B_{n}(M)\right] \longrightarrow \widehat{\mathcal{A}}_{n} \rtimes \mathbb{Z}\left[H_{n}\right]=\widehat{\mathcal{A}}_{n} \otimes \mathbb{Z}\left[H_{n}\right]
$$

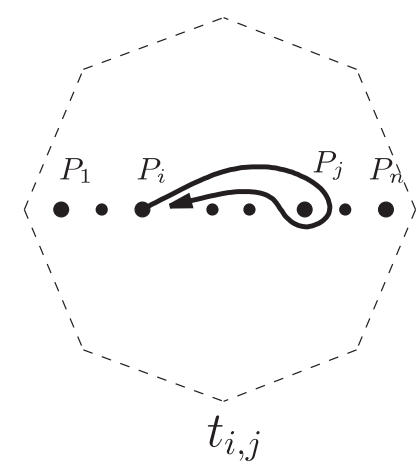

FigurE 7 . The braid $t_{i, j}$.

Recall that, for all $\gamma \in \pi_{1}(M)$ and all $i \in\{1, \ldots, n\}$, we denote by $\widetilde{\gamma}_{(i)}$ the normal form of $\gamma$ over the generators $\left\{a_{i, 1}^{ \pm 1}, \ldots, a_{i, 2 g}^{ \pm 1}\right\}$ of $\pi_{1}\left(M, P_{i}\right)$. For $1 \leq i<j \leq n$, we write $t_{i, j}=t_{j, i}=T_{i, j} T_{i, j-1}^{-1}$, which is the braid drawn in Figure 7 . These braids are the classical generators of $P B_{n}(D)$. Then, for $i \neq j$, we denote by $f_{i, j, \gamma}$ the element $\widetilde{\gamma}_{(i)} t_{i, j} \widetilde{\gamma}_{(i)}^{-1}$ of $B_{n}(M)$. From Lemma 2.5 it follows that $F_{(n+1)-i}$ is the free group freely generated by

$$
\mathcal{F}_{i, n}=\left\{f_{i, j, \gamma} ; j=i+1, \ldots, n, \quad \gamma \in \pi_{1}(M)\right\} .
$$

Moreover, it is shown in Subsection 2.3 that $K_{n}=F_{n} \rtimes\left(F_{n-1} \rtimes\left(\cdots \rtimes\left(F_{3} \rtimes F_{2}\right) \cdots\right)\right)$. So, every element $k \in K_{n}$ can be uniquely written in the form $k=k_{1} \cdots k_{n-1}$, where $k_{i}$ is a reduced word over $\mathcal{F}_{i, n} \cup \mathcal{F}_{i, n}^{-1}$. Now, for $i \in\{1, \ldots, n-1\}$, there is an injective multiplicative homomorphism $u_{i}: F_{(n+1)-i} \rightarrow \mathbb{Z} \llbracket t_{i, j, \gamma} \rrbracket$, where $\mathbb{Z} \llbracket t_{i, j, \gamma} \rrbracket$ denotes the ring of non-commutative formal power series over non-commutative variables $t_{i, j, \gamma}$, defined by

$$
\begin{aligned}
& u_{i}\left(f_{i, j, \gamma}\right)=1+t_{i, j, \gamma}, \\
& u_{i}\left(f_{i, j, \gamma}^{-1}\right)=1-t_{i, j, \gamma}+t_{i, j, \gamma}^{2}-\cdots .
\end{aligned}
$$

This well-known homomorphism is called the Magnus expansion of $F_{(n+1)-i}$ (see [12 $)$. We denote by $v_{i}$ the composition of $u_{i}$ with the canonical projection $\mathbb{Z} \llbracket t_{i, j, \gamma} \rrbracket \rightarrow$ $\widehat{\mathcal{A}}_{n}$, and we finally define the linear map $v: \mathbb{Z}\left[K_{n}\right] \rightarrow \widehat{\mathcal{A}}_{n}$ by

$$
v(k)=v_{1}\left(k_{1}\right) v_{2}\left(k_{2}\right) \cdots v_{n-1}\left(k_{n-1}\right),
$$

where $k=k_{1} k_{2} \cdots k_{n-1}$ is the decomposition of $k \in K_{n}$ defined above. 
3.2. The isomorphism $\chi_{1}: \mathcal{A}_{n} \rightarrow \mathcal{U} \operatorname{gr}\left(K_{n}\right)$. The goal of this subsection is to prove the following.

Proposition 3.1. There is a well-defined isomorphism $\chi_{1}: \mathcal{A}_{n} \rightarrow \mathcal{U} \operatorname{gr}\left(K_{n}\right)$ of $\mathbb{Z}$ algebras which sends $t_{i, j, \gamma}$ to $f_{i, j, \gamma}$ for all $i, j \in\{1, \ldots, n\}, i \neq j$, and all $\gamma \in \pi_{1}(M)$.

Consider the graded Lie algebra $L_{n}$ given by the following presentation:

- Generators: $\left\{t_{i, j, \gamma} ; 1 \leq i, j \leq n, i \neq j, \gamma \in \pi_{1}(M)\right\}$.

- Relations:

(L1) $t_{i, j, \gamma}=t_{j, i, \gamma^{-1}}, \quad$ for all $i, j \in\{1, \ldots, n\}, i \neq j$, and all $\gamma \in \pi_{1}(M)$,

(L2) $\left[t_{i, j, \gamma}, t_{k, l, \delta}\right]=0, \quad$ for all distinct $i, j, k, l \in\{1, \ldots, n\}$ and all $\gamma, \delta \in$ $\pi_{1}(M)$,

(L3) $\left[t_{i, j, \gamma}, t_{j, k, \delta}+t_{i, k,(\gamma \delta)}\right]=0$, for all distinct $i, j, k \in\{1, \ldots, n\}$ and all $\gamma, \delta \in \pi_{1}(M)$,

where [_, - ] denotes the Lie bracket.

One has $\mathcal{U} L_{n}=\mathcal{A}_{n}$, so, in order to prove Proposition [3.1, it suffices to prove the following.

Proposition 3.2. There is a well-defined Lie algebra isomorphism $\psi_{n}: L_{n} \longrightarrow$ $\operatorname{gr}\left(K_{n}\right)$ which sends $t_{i, j, \gamma}$ to $f_{i, j, \gamma}$ for all $i, j \in\{1, \ldots, n\}, i \neq j$, and all $\gamma \in \pi_{1}(M)$.

The following Lemmas 3.3 to 3.7 are preliminary results to the proof of Proposition 3.2

Lemma 3.3. Let $\omega$ be a word over $\Omega^{ \pm 1}$. Then there exists $W_{\omega} \in\left(K_{n}\right)_{2}=\left[K_{n}, K_{n}\right]$ such that

$$
\omega_{(j)} t_{i, j} \omega_{(j)}^{-1}=\left(\omega_{(i)}^{-1} t_{i, j} \omega_{(i)}\right) W_{\omega} .
$$

Proof. We can suppose, without loss of generality, that $i<j$. Suppose first that $\omega$ is a single letter. If $\omega_{(j)}=a_{j, r}$ and $r$ is odd, then one can easily show by drawing the braids that the following equality holds in $P B_{n}(M)$ :

$$
a_{j, r} t_{i, j} a_{j, r}^{-1}=\left(t_{i, j-1} \cdots t_{i, i+1}\right) a_{i, r}^{-1} t_{i, j} a_{i, r}\left(t_{i, i+1}^{-1} \cdots t_{i, j-1}^{-1}\right) .
$$

Hence

$$
a_{j, r} t_{i, j} a_{j, r}^{-1}=\left(a_{i, r}^{-1} t_{i, j} a_{i, r}\right) W_{\omega}
$$

where

$$
W_{\omega}=\left[a_{i, r}^{-1} t_{i, j}^{-1} a_{i, r}, t_{i, j-1} \cdots t_{i, i+1}\right] \in\left(K_{n}\right)_{2} .
$$

If $\omega_{(j)}=a_{j, r}$ and $r$ is even, then one has

$$
a_{j, r} t_{i, j} a_{j, r}^{-1}=a_{i, r}^{-1}\left(t_{i, i+1}^{-1} \cdots t_{i, j-1}^{-1} t_{i, j} \cdots t_{i, i+1}\right) a_{i, r} .
$$

Therefore,

$$
a_{j, r} t_{i, j} a_{j, r}^{-1}=\left(a_{i, r}^{-1} t_{i, j} a_{i, r}\right) W_{\omega},
$$

where

$$
W_{\omega}=\left[a_{i, r}^{-1} t_{i, j}^{-1} a_{i, r}, a_{i, r}^{-1}\left(t_{i, i+1}^{-1} \cdots t_{i, j-1}^{-1}\right) a_{i, r}\right] \in\left(K_{n}\right)_{2} .
$$

The computations for $\omega_{(j)}=a_{j, r}^{-1}$ are the same as for $\omega_{(j)}=a_{j, r}$, interchanging the case $r$ odd with the case $r$ even. 
Suppose now that $\omega$ is a word of length $k>1$, and that the result is true for words of length less than $k$. We write $\omega=\alpha \beta$, with $|\alpha|,|\beta|<k$. Consider

$$
\begin{aligned}
& W^{\prime}=\alpha_{(j)}^{-1} W_{\beta} \alpha_{(j)}, \\
& W^{\prime \prime}=\left[\beta_{(i)}^{-1} \alpha_{(j)} t_{i, j}^{-1} \alpha_{(j)}^{-1} \beta_{(i)},\left[\alpha_{(j)}, \beta_{(i)}^{-1}\right]\right] W^{\prime}, \\
& W=\beta_{(i)}^{-1} W_{\alpha} \beta_{(i)} W^{\prime \prime} .
\end{aligned}
$$

The hypothesis $i \neq j$ implies that $\left[\alpha_{(j)}, \beta_{(i)}^{-1}\right] \in K_{n}$. Furthermore, both $K_{n}$ and $\left(K_{n}\right)_{2}$ are normal subgroups of $P B_{n}(M)$; thus $W \in\left(K_{n}\right)_{2}$. Finally, a direct calculation shows that:

$$
\omega_{(j)} t_{i, j} \omega_{(j)}^{-1}=\left(\omega_{(i)}^{-1} t_{i, j} \omega_{(i)}\right) W
$$

as we wanted to show.

Lemma 3.4. Let $i, j \in\{2, \ldots, n\}, i \neq j$, let $\gamma \in \pi_{1}(M)$, and let $\omega$ be a word over $\Omega^{ \pm 1}$. Then there exists $W \in\left(K_{n}\right)_{2}$ such that

$$
f_{i, j, \gamma} \omega_{(1)} f_{i, j, \gamma}^{-1}=W \omega_{(1)} .
$$

Proof. Recall the epimorphism $\varrho: P B_{n}(M) \longrightarrow P B_{n-1}(M)$. Since one has $\varrho\left(\widetilde{\gamma}_{(i)}^{-1} \omega_{(1)} \widetilde{\gamma}_{(i)}\right)=1$, then one can write $\widetilde{\gamma}_{(i)}^{-1} \omega_{(1)} \widetilde{\gamma}_{(i)}=b$, where $b$ is a word over $\mathcal{B}=\left\{a_{1,1}^{ \pm 1}, \ldots, a_{1,2 g}^{ \pm 1}, t_{1,2}^{ \pm 1}, \ldots, t_{1, n}^{ \pm 1}\right\}$.

By drawing the braids, one sees that $t_{i, j} a_{1, r}^{ \pm 1} t_{i, j}^{-1}=a_{1, r}^{ \pm 1}$, for $r=1, \ldots, 2 g$. Moreover, since $t_{i, j}, t_{1, k} \in K_{n}$, one has $t_{i, j} t_{1, k}^{ \pm 1} t_{i, j}^{-1}=W t_{1, k}^{ \pm 1}$, where $W \in\left(K_{n}\right)_{2}$, for all $k=2, \ldots, n$. Therefore, since $b$ is a word over $\mathcal{B}$, one has: $t_{i, j} b t_{i, j}^{-1}=W_{b} b$, where $W_{b} \in\left(K_{n}\right)_{2}$. Hence,

$$
\begin{aligned}
f_{i, j, \gamma} \omega_{(1)} f_{i, j, \gamma}^{-1} & =\left(\widetilde{\gamma}_{(i)} t_{i, j} \widetilde{\gamma}_{(i)}^{-1}\right) \omega_{(1)}\left(\widetilde{\gamma}_{(i)} t_{i, j}^{-1} \widetilde{\gamma}_{(i)}^{-1}\right) \\
& =\widetilde{\gamma}_{(i)} t_{i, j} b t_{i, j}^{-1} \widetilde{\gamma}_{(i)}^{-1} \\
& =\widetilde{\gamma}_{(i)} W_{b} b \widetilde{\gamma}_{(i)}^{-1} \\
& =W \widetilde{\gamma}_{(i)} b \widetilde{\gamma}_{(i)}^{-1} \\
& =W \omega_{(1)},
\end{aligned}
$$

where $W=\widetilde{\gamma}_{(i)} W_{b} \widetilde{\gamma}_{(i)}^{-1} \in\left(K_{n}\right)_{2}$, as we wanted to show.

Lemma 3.5. Let $\omega$ be a word over $\Omega^{ \pm 1}$, and let $i, j, k \in\{1, \ldots, n\}$, all distinct. Then there exists $W \in\left(K_{n}\right)_{2}$ such that

$$
\omega_{(i)} t_{j, k} \omega_{(i)}^{-1}=W t_{j, k}
$$

Proof. Clearly, it suffices to show the lemma when $\omega$ is a single letter. Besides, we can suppose that $j<k$. Then the result is a consequence of the following relations in $P B_{n}(M)$ :

$$
\begin{array}{lll}
a_{i, r} t_{j, k} a_{i, r}^{-1}=t_{j, k}, & a_{i, r}^{-1} t_{j, k} a_{i, r}=t_{j, k}, & \text { if } i<j \text { or } i>k, \\
a_{i, r} t_{j, k} a_{i, r}^{-1}=t_{j, i}^{-1} t_{j, k} t_{j, i}, & a_{i, r}^{-1} t_{j, k} a_{i, r}=\alpha t_{j, k} \alpha^{-1}, & \text { if } j<i<k, r \text { odd, } \\
a_{i, r} t_{j, k} a_{i, r}^{-1}=\alpha t_{j, k} \alpha^{-1}, & a_{i, r}^{-1} t_{j, k} a_{i, r}=t_{j, i}^{-1} t_{j, k} t_{j, i}, & \text { if } j<i<k, r \text { even, }
\end{array}
$$


where $\alpha=\left(a_{j, r}^{-1} t_{j, j+1}^{-1} \cdots t_{j, i-1}^{-1} t_{j, i} \cdots t_{j, j+1} a_{j, r}\right) \in K_{n}$. These relations can be easily verified by drawing pictures.

Lemma 3.6. Let $i, j, k, l \in\{1, \ldots, n\}$, all distinct. Then

$$
\begin{aligned}
& {\left[t_{i, j}, t_{k, l}\right] \equiv 0 \quad\left(\bmod \left(K_{n}\right)_{3}\right),} \\
& {\left[t_{i, j}, t_{i, k}\right] \equiv\left[t_{j, k}, t_{i, j}\right] \quad\left(\bmod \left(K_{n}\right)_{3}\right) .}
\end{aligned}
$$

Proof. This lemma follows from the well-known congruences

$$
\begin{aligned}
& {\left[t_{i, j}, t_{k, l}\right] \equiv 0 \quad\left(\bmod \left(P B_{n}(D)\right)_{3}\right)} \\
& {\left[t_{i, j}, t_{i, k}\right] \equiv\left[t_{j, k}, t_{i, j}\right] \quad\left(\bmod \left(P B_{n}(D)\right)_{3}\right)}
\end{aligned}
$$

(see, for example, [4]), together with the inclusion $\left(P B_{n}(D)\right)_{3} \subset\left(K_{n}\right)_{3}$.

Lemma 3.7. There is a well-defined Lie algebra homomorphism $\psi_{n}: L_{n} \longrightarrow$ $\operatorname{gr}\left(K_{n}\right)$ which sends $t_{i, j, \gamma}$ to $f_{i, j, \gamma}$ for all $i, j \in\{1, \ldots, n\}, i \neq j$, and all $\gamma \in \pi_{1}(M)$.

Proof. We have to show that the following congruences hold:

(R1)

$$
\begin{array}{ll}
f_{i, j, \gamma} \equiv f_{j, i, \gamma^{-1}} & \left(\bmod \left(K_{n}\right)_{2}\right), \\
& \text { for all } i, j \in\{1, \ldots, n\} \\
& i \neq j, \text { and all } \gamma \in \pi_{1}(M) \\
{\left[f_{i, j, \gamma}, f_{k, l, \delta}\right] \equiv 0 \quad\left(\bmod \left(K_{n}\right)_{3}\right),} & \text { for all distinct } \\
& i, j, k, l \in\{1, \ldots, n\} \\
& \text { and all } \gamma, \delta \in \pi_{1}(M)
\end{array}
$$

(R2) $\left[f_{i, j, \gamma}, f_{k, l, \delta}\right] \equiv 0$

(R3) $\left[f_{i, j, \gamma}, f_{j, k, \delta}\right] \equiv\left[f_{i, k,(\gamma \delta)}, f_{i, j, \gamma}\right]\left(\bmod \left(K_{n}\right)_{3}\right)$, for all distinct

$$
\begin{aligned}
& i, j, k \in\{1, \ldots, n\} \\
& \text { and all } \gamma, \delta \in \pi_{1}(M) .
\end{aligned}
$$

Notice that (R1) follows from Lemma 3.3. So, it remains to prove (R2) and (R3). We argue by induction on $n$. The conditions (R2) and (R3) being empty if $n=2$, we may assume that $n>2$, that (R2) holds if $i, j, k, l \in\{2, \ldots, n\}$ (by induction), and that (R3) holds if $i, j, k \in\{2, \ldots, n\}$ (by induction).

We now prove (R2) for $k=1$. By Lemma 3.4, there exists $W_{1} \in\left(K_{n}\right)_{2}$ such that

$$
f_{i, j, \gamma} \widetilde{\delta}_{(1)} f_{i, j, \gamma}^{-1}=W_{1} \widetilde{\delta}_{(1)}
$$

Also, by Lemma 3.5, there exists $W_{2} \in\left(K_{n}\right)_{2}$ such that

$$
\widetilde{\gamma}_{(i)}^{-1} t_{1, l} \widetilde{\gamma}_{(i)}=W_{2} t_{1, l}
$$


Then

$$
\begin{aligned}
f_{i, j, \gamma} f_{1, l, \delta} f_{i, j, \gamma}^{-1} & =\left(f_{i, j, \gamma} \widetilde{\delta}_{(1)} f_{i, j, \gamma}^{-1}\right) f_{i, j, \gamma} t_{1, l} f_{i, j, \gamma}^{-1}\left(f_{i, j, \gamma} \widetilde{\delta}_{(1)}^{-1} f_{i, j, \gamma}^{-1}\right) \\
& =W_{1}\left(\widetilde{\delta}_{(1)} f_{i, j, \gamma} t_{1, l} f_{i, j, \gamma}^{-1} \widetilde{\delta}_{(1)}^{-1}\right) W_{1}^{-1} \\
& \equiv \widetilde{\delta}_{(1)} f_{i, j, \gamma} t_{1, l} f_{i, j, \gamma}^{-1} \widetilde{\delta}_{(1)}^{-1} \quad\left(\bmod \left(K_{n}\right)_{3}\right) \\
& =\widetilde{\delta}_{(1)}\left(\widetilde{\gamma}_{(i)} t_{i, j} \widetilde{\gamma}_{(i)}^{-1}\right) t_{1, l}\left(\widetilde{\gamma}_{(i)} t_{i, j}^{-1} \widetilde{\gamma}_{(i)}^{-1}\right) \widetilde{\delta}_{(1)}^{-1} \\
& =\widetilde{\delta}_{(1)} \widetilde{\gamma}_{(i)} t_{i, j} W_{2} t_{1, l} t_{i, j}^{-1} \widetilde{\gamma}_{(i)}^{-1} \widetilde{\delta}_{(1)}^{-1} \quad\left(\bmod \left(K_{n}\right)_{3}\right) \\
& \equiv \widetilde{\delta}_{(1)} \widetilde{\gamma}_{(i)} W_{2} t_{i, j} t_{1, l} t_{i, j}^{-1} \widetilde{\gamma}_{(i)}^{-1} \widetilde{\delta}_{(1)}^{-1} \quad\left(\bmod \left(K_{n}\right)_{3}\right) \quad \quad(\text { Lemma 3.6) }) \\
& \equiv \widetilde{\delta}_{(1)} \widetilde{\gamma}_{(i)} W_{2} t_{1, l} \widetilde{\gamma}_{(i)}^{-1} \widetilde{\delta}_{(1)}^{-1} \quad(\bmod \\
& =\widetilde{\delta}_{(1)} t_{1, l} \widetilde{\delta}_{(1)}^{-1}=f_{1, l, \delta} .
\end{aligned}
$$

Therefore, (R2) holds for $k=1$.

The congruence (R2) holds for either $i=1$, or $j=1$, or $l=1$, because of the above case and the relation (R1).

We now prove (R3) for $i=1$. By Lemma 3.4 there exists $W_{1} \in\left(K_{n}\right)_{2}$ such that

$$
f_{j, k, \delta} \widetilde{\gamma}_{(1)} f_{j, k, \delta}^{-1}=W_{1} \widetilde{\gamma}_{(1)} .
$$

Also, by Lemma 3.3, there exists $W_{2} \in\left(K_{n}\right)_{2}$ such that

$$
\widetilde{\delta}_{(j)}^{-1} t_{1, j} \widetilde{\delta}_{(j)}=\widetilde{\delta}_{(1)} t_{1, j} \widetilde{\delta}_{(1)}^{-1} W_{2}
$$

Then

$$
\begin{aligned}
f_{j, k, \delta} f_{1, j, \gamma} f_{j, k, \delta}^{-1} & =\left(f_{j, k, \delta} \widetilde{\gamma}_{(1)} f_{j, k, \delta}^{-1}\right)\left(f_{j, k, \delta} t_{1, j} f_{j, k, \delta}^{-1}\right)\left(f_{j, k, \delta} \widetilde{\gamma}_{(1)}^{-1} f_{j, k, \delta}^{-1}\right) \\
& =W_{1}\left(\widetilde{\gamma}_{(1)} f_{j, k, \delta} t_{1, j} f_{j, k, \delta}^{-1} \widetilde{\gamma}_{(1)}^{-1}\right) W_{1}^{-1} \\
& \equiv \widetilde{\gamma}_{(1)} f_{j, k, \delta} t_{1, j} f_{j, k, \delta}^{-1} \widetilde{\gamma}_{(1)}^{-1}\left(\bmod \left(K_{n}\right)_{3}\right) \\
& =\widetilde{\gamma}_{(1)}\left(\widetilde{\delta}_{(j)} t_{j, k} \widetilde{\delta}_{(j)}^{-1}\right) t_{1, j}\left(\widetilde{\delta}_{(j)} t_{j, k}^{-1} \widetilde{\delta}_{(j)}^{-1}\right) \widetilde{\gamma}_{(1)}^{-1} \\
& =\widetilde{\gamma}_{(1)} \widetilde{\delta}_{(j)} t_{j, k} \widetilde{\delta}_{(1)} t_{1, j} \widetilde{\delta}_{(1)}^{-1} W_{2} t_{j, k}^{-1} \widetilde{\delta}_{(j)}^{-1} \widetilde{\gamma}_{(1)}^{-1} \\
& \equiv \widetilde{\gamma}_{(1)} \widetilde{\delta}_{(j)}\left(t_{j, k} \widetilde{\delta}_{(1)} t_{1, j} \widetilde{\delta}_{(1)}^{-1} t_{j, k}^{-1}\right) W_{2} \widetilde{\delta}_{(j)}^{-1} \widetilde{\gamma}_{(1)}^{-1} \quad\left(\bmod \left(K_{n}\right)_{3}\right) \\
& =\widetilde{\gamma}_{(1)} \widetilde{\delta}_{(j)}\left[t_{j, k}, \widetilde{\delta}_{(1)} t_{1, j} \widetilde{\delta}_{(1)}^{-1}\right]\left(\widetilde{\delta}_{(1)} t_{1, j} \widetilde{\delta}_{(1)}^{-1}\right) W_{2} \widetilde{\delta}_{(j)}^{-1} \widetilde{\gamma}_{(1)}^{-1} \\
& =\widetilde{\gamma}_{(1)} \widetilde{\delta}_{(j)}\left[t_{j, k}, \widetilde{\delta}_{(1)} t_{1, j} \widetilde{\delta}_{(1)}^{-1}\right] \widetilde{\delta}_{(j)}^{-1} t_{1, j} \widetilde{\gamma}_{(1)}^{-1} \cdot
\end{aligned}
$$

Since $\widetilde{\delta}_{(1)}$ commutes with $t_{j, k}$, it follows that

$$
\begin{aligned}
& f_{j, k, \delta} f_{1, j, \gamma} f_{j, k, \delta}^{-1} \equiv \widetilde{\gamma}_{(1)} \widetilde{\delta}_{(j)} \widetilde{\delta}_{(1)}\left[t_{j, k}, t_{1, j}\right] \widetilde{\delta}_{(1)}^{-1} \widetilde{\delta}_{(j)}^{-1} t_{1, j} \widetilde{\gamma}_{(1)}^{-1} \quad\left(\bmod \left(K_{n}\right)_{3}\right) \\
& \equiv \widetilde{\gamma}_{(1)} \widetilde{\delta}_{(j)} \widetilde{\delta}_{(1)}\left[t_{1, j}, t_{1, k}\right] \widetilde{\delta}_{(1)}^{-1} \widetilde{\delta}_{(j)}^{-1} t_{1, j} \widetilde{\gamma}_{(1)}^{-1} \quad\left(\bmod \left(K_{n}\right)_{3}\right) \\
& \text { (by Lemma 3.6) } \\
& =\widetilde{\gamma}_{(1)}\left[\widetilde{\delta}_{(j)}, \widetilde{\delta}_{(1)}\right]\left(\widetilde{\delta}_{(1)} \widetilde{\delta}_{(j)}\left[t_{1, j}, t_{1, k}\right] \widetilde{\delta}_{(j)}^{-1} \widetilde{\delta}_{(1)}^{-1}\right)\left[\widetilde{\delta}_{(j)}, \widetilde{\delta}_{(1)}\right]^{-1} t_{1, j} \widetilde{\gamma}_{(1)}^{-1}
\end{aligned}
$$


Notice that $\left[\widetilde{\delta}_{(j)}, \widetilde{\delta}_{(1)}\right] \in K_{n}$ and $\left[t_{1, j}, t_{1, k}\right] \in\left(K_{n}\right)_{2}$; thus

$$
\begin{aligned}
f_{j, k, \delta} f_{1, j, \gamma} f_{j, k, \delta}^{-1} & \equiv \widetilde{\gamma}_{(1)}\left(\widetilde{\delta}_{(1)} \widetilde{\delta}_{(j)}\left[t_{1, j}, t_{1, k}\right] \widetilde{\delta}_{(j)}^{-1} \widetilde{\delta}_{(1)}^{-1}\right) t_{1, j} \widetilde{\gamma}_{(1)}^{-1} \quad\left(\bmod \left(K_{n}\right)_{3}\right) \\
& \equiv \widetilde{\gamma}_{(1)}\left[t_{1, j}, \widetilde{\delta}_{(1)} t_{1, k} \widetilde{\delta}_{(1)}^{-1}\right] t_{1, j} \widetilde{\gamma}_{(1)}^{-1} \quad\left(\bmod \left(K_{n}\right)_{3}\right) \\
& =\left[\widetilde{\gamma}_{(1)} t_{1, j} \widetilde{\gamma}_{(1)}^{-1}, \widetilde{\gamma}_{(1)} \widetilde{\delta}_{(1)} t_{1, k} \widetilde{\delta}_{(1)}^{-1} \widetilde{\gamma}_{(1)}^{-1}\right]\left(\widetilde{\gamma}_{(1)} t_{1, j} \widetilde{\gamma}_{(1)}^{-1}\right) .
\end{aligned}
$$

Let $h=\widetilde{\gamma}_{(1)} \widetilde{\delta}_{(1)} \widetilde{\gamma \delta}_{(1)}^{-1}$. One has $h \in K_{n}$; thus

$$
\begin{array}{rlr}
f_{j, k, \delta} f_{1, j, \gamma} f_{j, k, \delta}^{-1} & \equiv\left[\widetilde{\gamma}_{(1)} t_{1, j} \widetilde{\gamma}_{(1)}^{-1}, h \widetilde{(\gamma \delta)_{(1)}} t_{1, k} \widetilde{(\gamma \delta)_{(1)}^{-1}} h^{-1}\right] & \left(\widetilde{\gamma}_{(1)} t_{1, j} \widetilde{\gamma}_{(1)}^{-1}\right) \\
& =\left[f_{1, j, \gamma}, k f_{1, k,(\gamma \delta)} k^{-1}\right] f_{1, j, \gamma} & \left(\bmod \left(K_{n}\right)_{3}\right) \\
& \equiv\left[f_{1, j, \gamma}, f_{1, k,(\gamma \delta)}\right] f_{1, j, \gamma} & \left(\bmod \left(K_{n}\right)_{3}\right) .
\end{array}
$$

This proves that (R3) holds for $i=1$.

For $j=1$, (R3) holds because of the above case and (R1), since one has

$$
\left[f_{i, 1, \gamma}, f_{1, k, \delta}\right] \equiv\left[f_{1, i, \gamma^{-1}}, f_{1, k, \delta}\right] \equiv\left[f_{i, k,(\gamma \delta)}, f_{1, i, \gamma^{-1}}\right] \equiv\left[f_{i, k,(\gamma \delta)}, f_{i, 1, \gamma}\right] .
$$

Finally, (R3) also holds for $k=1$, since in this case

$$
\left[f_{i, j, \gamma}, f_{j, 1, \delta}\right] \equiv\left[f_{j, i, \gamma^{-1}}, f_{j, 1, \delta}\right] \equiv\left[f_{i, 1,(\gamma \delta)}, f_{j, i, \gamma^{-1}}\right] \equiv\left[f_{i, 1,(\gamma \delta)}, f_{i, j, \gamma}\right] .
$$

Proof of Proposition 3.2. It suffices to prove that the homomorphism $\psi_{n}: L_{n} \rightarrow$ $\operatorname{gr}\left(K_{n}\right)$ of Lemma 3.7] is an isomorphism. We argue by induction on $n$.

For $n=2, \quad K_{2}=F_{2}$ is a free group freely generated by $\mathcal{F}_{1,2}=\left\{f_{1,2, \gamma} ; \gamma \in\right.$ $\left.\pi_{1}(M)\right\}$, so $\operatorname{gr}\left(K_{2}\right)$ is the free Lie algebra generated by $\mathcal{F}_{1,2}$. On the other hand, $L_{2}$ is by definition the free Lie algebra generated by $\left\{t_{1,2, \gamma} ; \gamma \in \pi_{1}(M)\right\}$. Therefore, $\psi_{2}$ is a Lie algebra isomorphism.

Suppose now that $\psi_{m}$ is an isomorphism for $m<n$. Recall that $K_{n}=F_{n} \rtimes K_{n-1}$, and that we have the exact sequence

$$
\begin{aligned}
& 1 \longrightarrow F_{n} \longrightarrow K_{n} \quad \stackrel{\varrho}{\longrightarrow} K_{n-1} \longrightarrow 1 \text {, } \\
& f_{1, j, \gamma} \longmapsto \begin{array}{c}
f_{1, j, \gamma} \\
f_{i+1, j+1, \gamma}
\end{array}
\end{aligned}
$$

Since $K_{n-1}$ acts trivially on the abelianization of $F_{n}$, we can apply the result in [7] which claims that the associated graded sequence of Lie algebras is exact, that is,

$$
1 \longrightarrow \operatorname{gr}\left(F_{n}\right) \stackrel{i}{\longrightarrow} \operatorname{gr}\left(K_{n}\right) \stackrel{\operatorname{gr} \varrho}{\longrightarrow} \operatorname{gr}\left(K_{n-1}\right) \longrightarrow 1,
$$

where $i$ is the natural inclusion. Besides, since $\operatorname{gr}\left(F_{m}\right)$ is a free Lie algebra for all $m \geq 2$, the above sequence shows, by induction, that $\operatorname{gr}\left(K_{n}\right)$ is a free $\mathbb{Z}$-module. This fact will be used later on. 
Let us now define the following Lie algebra homomorphism:

$$
\begin{aligned}
\widetilde{\varrho}_{n}: \quad L_{n} & \longrightarrow L_{n-1}, \\
t_{1, j, \gamma} & \longmapsto 0, \\
t_{i+1, j+1, \gamma} & \longmapsto t_{i, j, \gamma} .
\end{aligned}
$$

Looking at the relations (L1), (L2) and (L3), we see that $\widetilde{\varrho}_{n}$ is a well-defined epimorphism of Lie algebras. We will denote $Q_{n}=\operatorname{ker} \widetilde{\varrho}_{n}$. In this way, we obtain the following commutative diagram:

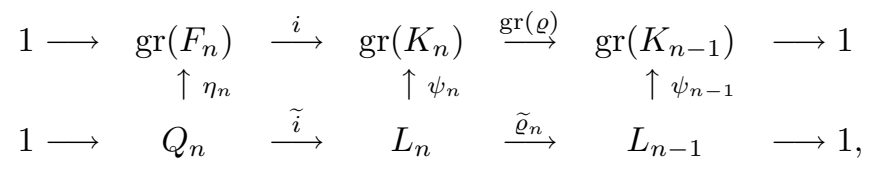

where $\eta_{n}$ is the restriction of $\psi_{n}$ to $Q_{n}$.

Notice that $t_{1, j, \gamma} \in Q_{n}$ for all $j=2, \ldots, n$ and all $\gamma \in \pi_{1}(M)$. Notice as well that $\eta_{n}\left(t_{1, j, \gamma}\right)=f_{1, j, \gamma}$, and that $\operatorname{gr}\left(F_{n}\right)$ is the free Lie algebra generated by $\mathcal{F}_{1, n}$. Therefore, if we show that $Q_{n}$ is generated (as a Lie algebra) by $\mathcal{B}_{1, n}=\left\{t_{1, j, \gamma} ; j=\right.$ $\left.2, \ldots, n ; \gamma \in \pi_{1}(M)\right\}$, then $Q_{n}$ will be the free Lie algebra generated by $\mathcal{B}_{1, n}$, and $\eta_{n}$ will be an isomorphism. In this case, since $\psi_{n-1}$ is an isomorphism by the induction hypothesis, $\psi_{n}$ will also be a Lie algebra isomorphism, as we want to show.

Let $l=\sum_{i=1}^{k} l_{i}$ be an element of $Q_{n}$, where each $l_{i}$ is a Lie bracket over the generators of $L_{n}$. We can decompose $l=\left(\sum_{i=1}^{r} l_{i}\right)+\left(\sum_{i=r+1}^{k} l_{i}\right)$, where $\left\{l_{1}, \ldots, l_{r}\right\}$ are the Lie brackets in which some $t_{1, j, \gamma}$ appears, and $\left\{l_{r+1}, \ldots, l_{k}\right\}$ are Lie brackets over $\left\{t_{i, j, \gamma} ; 2 \leq i<j \leq n, \gamma \in \pi_{1}(M)\right\}$.

For all $i=1, \ldots, r, l_{i} \in Q_{n}$; hence $\sum_{i=r+1}^{k} l_{i} \in Q_{n}$. But if $\widetilde{\varrho}_{n}\left(\sum_{i=r+1}^{k} l_{i}\right)=0$ in $L_{n-1}$, then $\sum_{i=r+1}^{k} l_{i}=0$ in $L_{n}$, since the relations in $L_{n-1}$ are the images by $\widetilde{\varrho}_{n}$ of the same relations in $L_{n}$ which involve no $t_{1, j, \gamma}$. Therefore, $l=\sum_{i=1}^{r} l_{i}$, where each $l_{i}$ contains some $t_{1, j, \gamma}$. We must then show that each $l_{i}$ may be written as a sum of brackets over $\mathcal{B}_{1, n}$.

If $l_{i}$ is a bracket of length 2 , the result is a direct consequence of (L1), (L2) and (L3). Suppose that the result is true for brackets of length $d-1$, and consider $l_{i}=[a, b]$, a bracket of length $d>2$. We can suppose that $a$ contains some $t_{1, j, \gamma}$, and by induction, that it is a bracket over $\mathcal{B}_{1, n}$.

If length $(a) \geq 2$, then $a=\left[a_{1}, a_{2}\right]$, where $a_{1}, a_{2}$ are brackets over $\mathcal{B}_{1, n}$. By the Jacoby identity,

$$
l_{i}=\left[\left[a_{1}, a_{2}\right], b\right]=-\left[\left[a_{2}, b\right], a_{1}\right]-\left[\left[b, a_{1}\right], a_{2}\right],
$$

where $\left[a_{2}, b\right]$ and $\left[b, a_{1}\right]$ can be written, by the induction hypothesis, as a sum of brackets over $\mathcal{B}_{1, n}$, so the result follows.

If length $(a)=1$, then length $(b) \geq 2$, so $b=\left[b_{1}, b_{2}\right]$. Hence,

$$
\left[a,\left[b_{1}, b_{2}\right]\right]=\left[b_{1},\left[b_{2}, a\right]\right]-\left[b_{2},\left[a, b_{1}\right]\right]
$$

and we reduce to the previous case. Therefore, $Q_{n}$ is generated by $\mathcal{B}_{1, n}$, and hence $\psi_{n}$ is a Lie algebra isomorphism. 
3.3. The isomorphism $\chi_{2}: \mathcal{U} \operatorname{gr}\left(K_{n}\right) \rightarrow \operatorname{gr}_{I} \mathbb{Z}\left[K_{n}\right]$. We start this subsection by stating a result due to Quillen.

Theorem 3.8 (Quillen [14]). Let $G$ be a group. Let $I=I(G)$ be the augmentation ideal of $\mathbb{Z}[G]$, let $\operatorname{gr}_{I} \mathbb{Z}[G]$ be the graded ring associated with the $I$-adic filtration, and let $G=G_{1} \supset G_{2} \supset \cdots \supset G_{i} \supset \cdots$ be the lower central series of $G$. Then the maps $\kappa_{i}: G_{i} \rightarrow I^{i}, g \mapsto g-1$, induce a surjective homomorphism $\kappa: \mathcal{U} \operatorname{gr}(G) \rightarrow \operatorname{gr}_{I} \mathbb{Z}[G]$ of $\mathbb{Z}$-algebras. Moreover, $\kappa \otimes \mathbb{Q}$ is an isomorphism of $\mathbb{Q}$-algebras.

Notice that, if $\operatorname{gr}(G)$ is a free $\mathbb{Z}$-module, so is $\mathcal{U} \operatorname{gr}(G)$. Thus we have

Corollary 3.9. If $\operatorname{gr}(G)$ is a free $\mathbb{Z}$-module, then the maps $\kappa_{i}: G_{i} \rightarrow I^{i}, g \mapsto g-1$, induce an isomorphism $\kappa: \mathcal{U} \operatorname{gr}(G) \rightarrow \operatorname{gr}_{I} \mathbb{Z}[G]$ of $\mathbb{Z}$-algebras.

Now, it is shown in the proof of Proposition 3.2 that $\operatorname{gr}\left(K_{n}\right)$ is a free $\mathbb{Z}$-module. So:

Proposition 3.10. There is a well-defined isomorphism $\chi_{2}: \mathcal{U} \operatorname{gr}\left(K_{n}\right) \rightarrow \operatorname{gr}_{I} \mathbb{Z}\left[K_{n}\right]$ which sends $f_{i, j, \gamma}$ to $f_{i, j, \gamma}-1$, for all $i, j \in\{1, \ldots, n\}, i \neq j$, and all $\gamma \in \pi_{1}(M)$.

3.4. $\operatorname{gr} v$ is the inverse of $\chi=\chi_{2} \circ \chi_{1}$. We have shown in Subsections 3.2 and 3.3 that there is a well-defined isomorphism $\chi=\chi_{2} \circ \chi_{1}: \mathcal{A}_{n} \longrightarrow \operatorname{gr}_{I} \mathbb{Z}\left[K_{n}\right]$ which sends $t_{i, j, \gamma}$ to $f_{i, j, \gamma}-1$ for all $i, j \in\{1, \ldots, n\}, i \neq j$, and all $\gamma \in \pi_{1}(M)$. We now prove the following.

Proposition 3.11. The homomorphism grv is the inverse of $\chi$. Hence it is an isomorphism of graded $\mathbb{Z}$-algebras.

Proof. We only need to prove that grv is the inverse of $\chi$ as a homomorphism of $\mathbb{Z}$ modules. For $d \geq 1$, let $\mathcal{A}_{n}^{(d)}=\widehat{\mathcal{A}}_{n}^{(\geq d)} / \widehat{\mathcal{A}}_{n}^{(\geq d+1)}$ be the submodule of $\mathcal{A}_{n}$ consisting of the homogeneous polynomials of degree $d$. Consider also $i, j, k, l \in\{1, \ldots, n\}$, where $i<j, k<l$ and $i<k$. By relations (L1), (L2) and (L3), seen as relations in the enveloping algebra $\mathcal{A}_{n}$ of $L_{n}$, one has

$t_{k, l, \delta} t_{i, j, \gamma}= \begin{cases}t_{i, j, \gamma} t_{k, l, \delta} & \text { if } i, j, k, l \text { all distinct } \\ t_{i, j, \gamma} t_{k, l, \delta}+t_{i, j, \gamma} t_{i, l,(\gamma \delta)}-t_{i, l,(\gamma \delta)} t_{i, j, \gamma} & \text { if } j=k, \\ t_{i, j, \gamma} t_{k, l, \delta}+t_{i, j, \gamma} t_{i, k,\left(\gamma \delta^{-1}\right)}-t_{i, k,\left(\gamma \delta^{-1}\right)} t_{i, j, \gamma} & \text { if } j=l .\end{cases}$

Therefore, a set of generators for $\mathcal{A}_{n}^{(d)}$ as a $\mathbb{Z}$-module consists on the elements of the form

$$
R=t_{i_{1}, j_{1}, \gamma_{1}} t_{i_{2}, j_{2}, \gamma_{2}} \cdots t_{i_{d}, j_{d}, \gamma_{d}},
$$

where $i_{1} \leq i_{2} \leq \cdots \leq i_{d}$ and $i_{k}<j_{k}$ for all $k=1, \ldots, d$. But

$$
\chi(R)=\left(f_{i_{1}, j_{1}, \gamma_{1}}-1\right)\left(f_{i_{2}, j_{2}, \gamma_{2}}-1\right) \cdots\left(f_{i_{d}, j_{d}, \gamma_{d}}-1\right),
$$

so, by definition of grv, and since $i_{1} \leq i_{2} \leq \cdots \leq i_{d}$, one has $\operatorname{gr} v(\chi(R))=R$. This is true for all $d \geq 1$, so it follows that $\operatorname{gr} v \circ \chi=\operatorname{id}_{\mathcal{A}_{n}}$. Hence, since $\chi$ is an isomorphism, grv is its inverse, as we wanted to show. 
This result implies the following.

Theorem 3.12. gru is an isomorphism of $\mathbb{Z}$-modules.

Proof. Recall that, by Proposition 2.2 the ideal $V_{d}=J^{d}$ of $\mathbb{Z}\left[B_{n}(M)\right]$ is isomorphic to $I_{n}^{d} \otimes \mathbb{Z}\left[H_{n}\right]$ via $\Phi$, for all $d \geq 0$. Moreover, since $\mathbb{Z}\left[H_{n}\right]$ is a free $\mathbb{Z}$-module, one has

$$
V_{d} / V_{d+1} \simeq\left(I_{n}^{d} / I_{n}^{d+1}\right) \otimes \mathbb{Z}\left[H_{n}\right]
$$

Hence, $\operatorname{gr}_{V} \mathbb{Z}\left[B_{n}(M)\right] \simeq\left(\operatorname{gr}_{I} \mathbb{Z}\left[K_{n}\right]\right) \otimes \mathbb{Z}\left[H_{n}\right]$ via $\operatorname{gr} \Phi$. Now, gru $=(\operatorname{gr} v \otimes \mathrm{id}) \circ$ $\operatorname{gr} \Phi$, and both $\operatorname{gr} \Phi$ and $\operatorname{gr} v \otimes$ id are isomorphisms of $\mathbb{Z}$-modules. Thus gru is an isomorphism of $\mathbb{Z}$-modules.

3.5. $\mathrm{gr} u$ is a homomorphism. In this subsection, we finish the proof of Theorem 1.3 by showing that gr $u$ is a homomorphism.

We start by defining an algebra structure on $\operatorname{gr}_{I} \mathbb{Z}\left[K_{n}\right] \otimes \mathbb{Z}\left[H_{n}\right]$. Consider the action of $B_{n}(M)$ on $K_{n}$ by conjugation: an element $b \in B_{n}(M)$ sends $k \in K_{n}$ to $b k b^{-1} \in K_{n}$. This action extends naturally to $\mathbb{Z}\left[K_{n}\right]$ and preserves the $I$-adic filtration, so it defines an action of $B_{n}(M)$ on $\operatorname{gr}_{I} \mathbb{Z}\left[K_{n}\right]$. This action restricted to $K_{n}$ becomes trivial, since if $k, k^{\prime} \in K_{n}$, then

$$
k\left(k^{\prime}-1\right) k^{-1}=k k^{\prime} k^{-1}-1=\left[k, k^{\prime}\right] k^{\prime}-1,
$$

so, in $\operatorname{gr}_{I} \mathbb{Z}\left[K_{n}\right]$

$$
k\left(k^{\prime}-1\right) k^{-1} \equiv\left(\left[k, k^{\prime}\right]-1\right) k^{\prime}+\left(k^{\prime}-1\right) \equiv\left(k^{\prime}-1\right) .
$$

Therefore, the action induced on $\operatorname{gr}_{I} \mathbb{Z}\left[K_{n}\right]$ by an element $b \in B_{n}(M)$ depends only on $\varphi(b) \in H_{n}$. Recall the set map section $\sigma: H_{n} \rightarrow B_{n}(M)$. Now, define the product in $\operatorname{gr}_{I} \mathbb{Z}\left[K_{n}\right] \otimes \mathbb{Z}\left[H_{n}\right]$ by

$$
\left(k_{1} \otimes \beta_{1}\right)\left(k_{2} \otimes \beta_{2}\right)=\left(k_{1} \sigma\left(\beta_{1}\right) k_{2} \sigma\left(\beta_{1}\right)^{-1}\right) \otimes \beta_{1} \beta_{2} .
$$

By the above discussion, this product does not depend on $\sigma$, and it endows $\operatorname{gr}_{I} \mathbb{Z}\left[K_{n}\right] \otimes \mathbb{Z}\left[H_{n}\right]$ with a $\mathbb{Z}$-algebra structure.

Now, in order to prove that $\operatorname{gr} u=(\operatorname{gr} v \otimes \mathrm{id}) \circ \operatorname{gr} \Phi$ is a homomorphism of graded $\mathbb{Z}$-algebras, we turn to prove that both $\operatorname{gr} \Phi$ and (gr $v \otimes$ id) are homomorphisms of graded $\mathbb{Z}$-algebras.

Lemma 3.13. $\operatorname{gr} \Phi: \operatorname{gr}_{V} \mathbb{Z}\left[B_{n}(M)\right] \rightarrow \operatorname{gr}_{I} \mathbb{Z}\left[K_{n}\right] \otimes \mathbb{Z}\left[H_{n}\right]$ is a homomorphism of graded $\mathbb{Z}$-algebras.

Proof. Let $b_{1}, b_{2} \in B_{n}(M)$. Write $\beta_{i}=\varphi\left(b_{i}\right)$ and $k_{i}=b_{i}(\sigma \circ \varphi)\left(b_{i}\right)^{-1}$ for $i=1,2$. Then

$$
\begin{gathered}
\operatorname{gr} \Phi\left(b_{1}\right) \operatorname{gr} \Phi\left(b_{2}\right)=\left(k_{1} \otimes \beta_{1}\right)\left(k_{2} \otimes \beta_{2}\right)=\left(k_{1} \sigma\left(\beta_{1}\right) k_{2} \sigma\left(\beta_{1}\right)^{-1}\right) \otimes \beta_{1} \beta_{2}, \\
\operatorname{gr} \Phi\left(b_{1} b_{2}\right)=\left(k_{1} \sigma\left(\beta_{1}\right) k_{2} \sigma\left(\beta_{2}\right) \sigma\left(\beta_{1} \beta_{2}\right)^{-1}\right) \otimes \beta_{1} \beta_{2} .
\end{gathered}
$$

So, in order to prove that $\operatorname{gr} \Phi\left(b_{1} b_{2}\right)=\operatorname{gr} \Phi\left(b_{1}\right) \operatorname{gr} \Phi\left(b_{2}\right)$, it suffices to show that

$$
\sigma\left(\beta_{1}\right) \sigma\left(\beta_{2}\right) \equiv \sigma\left(\beta_{1} \beta_{2}\right) \quad\left(\bmod V_{1}\right)
$$


But $\varphi\left(\sigma\left(\beta_{1}\right) \sigma\left(\beta_{2}\right)\right)=\beta_{1} \beta_{2}=\varphi\left(\sigma\left(\beta_{1} \beta_{2}\right)\right)$, and thus there exists $k \in K_{n}$ such that $\sigma\left(\beta_{1}\right) \sigma\left(\beta_{2}\right)=k \sigma\left(\beta_{1} \beta_{2}\right)$ with $k \in K_{n}$. Hence, in $\mathbb{Z}\left[B_{n}(M)\right]$,

$$
\sigma\left(\beta_{1}\right) \sigma\left(\beta_{2}\right)-\sigma\left(\beta_{1} \beta_{2}\right)=(k-1) \sigma\left(\beta_{1} \beta_{2}\right) \in V_{1},
$$

since $k-1 \in V_{1}$, as we wanted to show.

Lemma 3.14. gr $v \otimes \mathrm{id}: \operatorname{gr}_{I} \mathbb{Z}\left[K_{n}\right] \otimes \mathbb{Z}\left[H_{n}\right] \rightarrow \mathcal{A}_{n} \rtimes \mathbb{Z}\left[H_{n}\right]$ is a homomorphism of graded $\mathbb{Z}$-algebras.

Proof. Write $g=\operatorname{gr} v$ and $g^{\prime}=\operatorname{gr} v \otimes \mathrm{id}=g \otimes \mathrm{id}$, to simplify notation. Also, write $\beta_{1}^{\prime}=\sigma\left(\beta_{1}\right)$. We know that $g$ is a $\mathbb{Z}$-algebra isomorphism, so

$$
\begin{aligned}
g^{\prime}\left(\left(k_{1} \otimes \beta_{1}\right)\left(k_{2} \otimes \beta_{2}\right)\right) & =g^{\prime}\left(\left(k_{1} \beta_{1}^{\prime} k_{2} \beta_{1}^{\prime-1}\right) \otimes \beta_{1} \beta_{2}\right) \\
& =g\left(k_{1} \beta_{1}^{\prime} k_{2} \beta_{1}^{\prime-1}\right) \otimes \beta_{1} \beta_{2} \\
& =g\left(k_{1}\right) g\left(\beta_{1}^{\prime} k_{2} \beta_{1}^{\prime-1}\right) \otimes \beta_{1} \beta_{2} .
\end{aligned}
$$

On the other hand:

$$
\begin{aligned}
g^{\prime}\left(k_{1} \otimes \beta_{1}\right) g^{\prime}\left(k_{2} \otimes \beta_{2}\right) & =\left(g\left(k_{1}\right) \otimes \beta_{1}\right)\left(g\left(k_{2}\right) \otimes \beta_{2}\right) \\
& =g\left(k_{1}\right)\left(\beta_{1} g\left(k_{2}\right) \beta_{1}^{-1}\right) \otimes \beta_{1} \beta_{2} .
\end{aligned}
$$

Therefore, we need to show that, in $\mathcal{A}_{n}$,

$$
g\left(\sigma\left(\beta_{1}\right) k_{2} \sigma\left(\beta_{1}\right)^{-1}\right)=\beta_{1} g\left(k_{2}\right) \beta_{1}^{-1} .
$$

Since the action by conjugation does not depend on $\sigma$, we can assume that $\beta_{1}$ is a generator of $H_{n}$. Moreover, since $g$ is a homomorphism of $\mathbb{Z}$-algebras, it suffices to verify the above formula when $k_{2}$ is a generator of $\operatorname{gr}_{I} \mathbb{Z}\left[K_{n}\right]$ as a $\mathbb{Z}$-algebra, that is, when $k_{2}=f_{i, j, \gamma}-1, i<j$. Hence, it suffices to prove Lemma 3.15 below.

Lemma 3.15. In $\operatorname{gr}_{I} \mathbb{Z}\left[K_{n}\right]$ the following relations hold, for all $i, j, k \in\{1, \ldots, n\}$ and all $\gamma \in \pi_{1}(M)$ :

- $\sigma_{k} f_{i, j, \gamma} \sigma_{k}^{-1}=f_{s_{k}(i), s_{k}(j), \gamma}$, where $s_{k}$ is the transposition $(k k+1)$,

- $a_{k, r} f_{i, j, \gamma} a_{k, r}^{-1}=f_{i, j, \gamma}, \quad$ if $k \neq i, j$,

- $a_{i, r} f_{i, j, \gamma} a_{i, r}^{-1}=f_{i, j,\left(\omega_{r} \gamma\right)}$,

where $\left\{\sigma_{1}, \ldots, \sigma_{n-1}\right\}$ and $\left\{a_{i, r} ; 1 \leq i \leq n\right.$ and $\left.1 \leq r \leq 2 g\right\}$ are the braids described in Subsection 2.1.

Proof. The first equation is a consequence of the following relations in $B_{n}(M)$, which are easily verified:

$$
\begin{aligned}
& \sigma_{k} a_{i, r} \sigma_{k}^{-1}=\left\{\begin{array}{lll}
a_{i, r} & \text { if } k \neq i-1, i, & \\
a_{i+1, r} t_{i, i+1}^{-1} & \text { if } k=i & \text { and } r \text { is even, } \\
t_{i, i+1} a_{i+1, r} & \text { if } k=i & \text { and } r \text { is odd, } \\
t_{i-1, i} a_{i-1, r} & \text { if } k=i-1 & \text { and } r \text { is even, } \\
a_{i-1, r} t_{i-1, i}^{-1} & \text { if } k=i-1 & \text { and } r \text { is odd, }
\end{array}\right. \\
& \sigma_{k} t_{i, j} \sigma_{k}^{-1}= \begin{cases}t_{i-1, j} & \text { if } k=i-1 \\
t_{i, i+1} t_{i+1, j} t_{i, i+1}^{-1} & \text { if } k=i \\
t_{i, j-1} & \text { if } k=j-1 \\
t_{i, j}^{-1} t_{i, j+1} t_{i, j} & \text { if } k=j \\
t_{i, j} & \text { otherwise. }\end{cases}
\end{aligned}
$$


The second equation comes from Lemma [3.5, and from the following relations, where $i \neq k$ and we denote $b_{l, m}=a_{l, m}$ if $m$ is odd, and $b_{l, m}=a_{l, m}^{-1}$ if $m$ is even:

$$
b_{k, r} b_{i, s} b_{k, r}^{-1}= \begin{cases}t_{i, k}^{-1} b_{i, s} & \text { if } s<r \quad \text { and } i<k, \\ b_{i, s}\left(b_{i, r}^{-1} t_{i, k} b_{i, r}\right) & \text { if } s>r \quad \text { and } i<k, \\ b_{i, s}\left(b_{i, r}^{-1} t_{k, i}^{-1} b_{i, r}\right) & \text { if } s<r \quad \text { and } i>k, \\ t_{k, i} b_{i, s} & \text { if } s>r \quad \text { and } i>k, \\ b_{i, s} & \text { if } s=r .\end{cases}
$$

Indeed, in this case,

$$
b_{k, r} f_{i, j, \gamma} b_{k, r}^{-1} \equiv b_{k, r} \widetilde{\gamma}_{(i)} t_{i, j} \widetilde{\gamma}_{(i)}^{-1} b_{k, r}^{-1} \equiv \widetilde{\gamma}_{(i)} b_{k, r} t_{i, j} b_{k, r}^{-1} \widetilde{\gamma}_{(i)}^{-1},
$$

and by Lemma 3.5 , this is equivalent to $f_{i, j, \gamma}$.

Finally, the third equation is verified as follows:

$$
a_{i, r} f_{i, j, \gamma} a_{i, r}^{-1} \equiv a_{i, r} \widetilde{\gamma}_{(i)} t_{i, j} \widetilde{\gamma}_{(i)}^{-1} a_{i, r}^{-1} \equiv k \widetilde{{\left(\omega_{r} \gamma\right)_{(i)}}_{i, j}} t_{\left(\omega_{r} \gamma\right)_{(i)}}^{-1} k^{-1}
$$

where $k \in K_{n}$, so this is equivalent to $f_{i, j,\left(\omega_{r} \gamma\right)}$.

\section{ACKNOWLEDGEMENT}

We are grateful to Ştefan Papadima for stimulating conversations and suggestions which were the starting point of this work.

\section{REFERENCES}

1. J. C. Baez, Link invariants of finite type and perturbation theory, Lett. Math. Phys. 26 (1992), no. 1, 43-51. MR 93k:57006

2. D. Bar-Natan, On the Vassiliev knot invariants, Topology 34 (1995), no. 2, 423-472. MR 97d:57004

3. D. Bar-Natan, Vassiliev homotopy string links invariants, J. Knot Theory Ramifications 4 (1995), no. 1, 13-32. MR 96b:57004

4. D. Bar-Natan, Vassiliev and quantum invariants of braids, The interface of knots and physics (San Francisco, CA, 1995), 129-144, Proc. Sympos. Appl. Math., 51, Amer. Math. Soc., Providence, RI, 1996. MR 97b:57004

5. J. S. Birman, Braids, links and mapping class groups, Annals of Math. Studies 82, Princeton University Press, 1974. MR 51:11477

6. J. S. Birman, New points of view in knot theory, Bull. Amer. Math. Soc. 28 (1993), no. 2, 253-287. MR 94b:57007

7. M. Falk and R. Randell, The lower central series of a fiber type arrangement, Invent. Math. 82 (1985), no. 1, 77-88. MR 87c:32015b

8. R. H. Fox, Free differential calculus I: Derivation in the free group ring, Ann. of Math. $\mathbf{5 7}$ (1953), no. 3, 547-560. MR 14:843d

9. J. González-Meneses, New presentations of surface braid groups, J. Knot Theory Ramifications, 10 (2001), no. 3, 431-451. MR 2002b:20040

10. T. Kohno, Vassiliev invariants and de Rahm complex on the space of knots, Symplectic geometry and quantization (Sanda and Yokohama, 1993), 123-138, Contemp. Math. 179, Amer. Math. Soc., Providence, RI, 1994. MR 96g:57010

11. R. C. Lyndon and P. E. Schupp, Combinatorial group theory, Springer-Verlag, 1977. MR 58:28182

12. W. Magnus, A. Karras and D. Solitar, Combinatorial group theory: Presentations of groups in terms of generators and relations, Dover Publications Inc., New York, 1976. MR 54:10423

13. Ş. Papadima, The universal finite-type invariant for braids, with integer coefficients, Topology Appl. 118 (2002), no. 1-2, 169-185.

14. D. Quillen, On the associated graded ring of a group ring, J. Algebra 10 (1968) 411-418. MR 38:245 
15. J. P. Serre, Lie algebras and Lie groups, 1964 lectures given at Harvard University, Second edition, Lecture Notes in Math. 1500, Springer-Verlag, Berlin, 1992. MR 93h:17001

16. T. Stanford, Braid commutators and Vassiliev invariants, Pacific J. Math. 174 (1996), no. 1, 269-276. MR 97i:57008

17. V. A. Vassiliev, Cohomology of knot spaces, Theory of singularities and its applications, 23-69, Adv. Soviet Math. 1, Amer. Math. Soc., Providence, RI, 1990. MR 92a:57016

18. V. A. Vassiliev, Complements of discriminants of smooth maps: topology and applications, Trans. of Math. Mono. 98, Amer. Math. Soc., Providence, RI, 1992. MR 94i:57020

Departamento de Álgebra, Facultad de Matemáticas, Universidad de Sevilla, c/ Tarfia S/n, 41012 Sevilla, Spain

E-mail address: meneses@us.es

Université de Bourgogne, Laboratoire de Topologie, UMR 5584 du CNRS, B.P. 47870 , 21078 - Dijon Cedex, France

E-mail address: lparis@u-bourgogne.fr 